\title{
J. BRIANCON
}

A. Galligo
M. Granger

\section{Déformations équisingulières des germes de courbes gauches réduites}

Mémoires de la S. M. F. $2^{e}$ série, tome 1 (1980)

$<$ http://www.numdam.org/item?id=MSMF_1980_2_1_1_0>

@ C Mémoires de la S. M. F., 1980, tous droits réservés.

L'accès aux archives de la revue « Mémoires de la S. M. F. » (http://smf. emath.fr/Publications/Memoires/Presentation.html) implique l'accord avec les conditions générales d'utilisation (http://www.numdam.org/conditions). Toute utilisation commerciale ou impression systématique est constitutive d'une infraction pénale. Toute copie ou impression de ce fichier doit contenir la présente mention de copyright.

\section{NumDam}




\section{$\begin{array}{lllllllllllllllll}I & N & T & R & 0 & D & U & C & T & I & 0 & N\end{array}$}

L'objet de ce travail est de comparer les définitions d'équisingularité introduites par différents auteurs dans le cas des déformations plates de germes de courbes réduites. Nous faisons une synthèse de ce qui nous est connu sur le sujet et présentons un certain nombre d'exemples et contre-exemples.

Le problème de l'équisingularité a d'abord été posé et étudié par 0 . ZARISKI. Nous nous sommes inspiré des travaux dans cette direction de F. PHAM et $B$. TEISSIER [P-T ] J. STUTZ [St ],B. TEISSIER $\left[T_{2}\right]$ et surtout de R.0. BUCHWEISS et G.M. GREUEL [B-G] . C'est ce dernier article qui permet de parler du nombre $\mu$ de Milnor et de " $\mu$ constant" pour une famille de courbes réduites sans rester dans le cadre limité des courbes intersections complètes et donne le résultat topologique fondamental.

RAPPEL SUR L'EQUISINGULARITÉ DES GERMES DE COURBES PLANES.

Dans $\left[Z_{1}\right], 0$. ZARISKI définit la (a)-équivalence de deux germes de courbes planes, par récurrence sur le nombre d'éclatements nécessaires à la désingularisation Soient $C$ et $D$ deux germes de courbes planes de branches respectives $\gamma_{1}, \ldots, \gamma_{r}$ et $\delta_{1}, \ldots \delta_{r}$. Une bijection $\pi$ entre les branches est dite tangentiellement stable si : deux branches quelconques sont tangentes si et seulement si leurs images par $\pi$ sont tangentes. M induit alors une bijection entre les composantes tangentielles de $C$ et $D$.

$C$ et $D$ sont (a)-équivalentes $S^{\prime} i l$ existe une bijection $\pi$ tangentiellement stable entre leurs branches telle que :

- les multiplicités d'une branche $\gamma_{i}$ et de son image $\pi\left(\gamma_{j}\right)$ sont égales, - $\pi$ induit une (a)-équivalence entre les transformées propres des composantes tangentielles de $C$ et $D$ dans l'éclatement de l'origine. 
Dans $\left[\mathrm{z}_{3}\right], 0$. ZARISKI démontre, grâce à la théorie de la saturation d'une algèbre analytique locale, que deux courbes planes irreductibles sont (a)équivalentes si et seulement si elles ont les mêmes paires caractéristiques de Puiseux; puis dans $\left[z_{4}\right]$ il démontre que deux courbes planes sont (a)-équivalentes par $\pi$ si et seulement si pour tout $i, \gamma_{i}$ et $\pi\left(\gamma_{i}\right)$ ont les mêmes paires de Puiseux et pour tout $(i, j)$, les multiplicités d'intersection $\left(\gamma_{i} ; \gamma_{j}\right)$ et $\left(\pi\left(\gamma_{j}\right) ; \pi\left(\gamma_{j}\right)\right)$ sont égales.

D'après des résultats classiques $\left(\left[\mathrm{Br}_{r}\right],\left[\mathrm{Z}_{5}\right]\right.$ ) $i l$ en résulte alors que deux germes de courbes planes $C$ et $D$ sont (a)-équivalentes si et seulement si elles ont même type topologique : c'est-à-dire s'il existe deux voisinages ouverts de l'origine $U$ et $V$ dans $\mathbb{d}^{2}$ et $u$ homéomorphisme $h$ de $U$ sur $V$ envoyant $C$ sur $D$. Une déformation à base lisse de germes de courbe plane est dite équisingulière si deux fibres quelconques de la déformation sont (a)-équivalentesou topologiquement équivalentes.

Dans $\left[Z_{1}\right]$, 0. ZARiski démontre le critère discriminant : une déformation est équisingulière si et seulement si il existe une projection "permise" (voir $\left[\mathrm{Z}_{7}\right]$ ) pour laquelle le discriminant de la projection est équimultiple. Dans $\left[Z_{6}\right]$ il démontre aussi qu'une déformation est équisingulière si et seulement si elle vérifie les conditions (a) et (b) de Whitney, voir aussi

B. Teissier $\left[\mathrm{T}_{4}\right]$. Dans $\left[\mathrm{Le}_{2}\right]$ et [L-R] Lê DṸng TRÁNG et C.P. RAMANUJAM montrent par des arguments topologiques qu'une déformation de courbe plane dont la fibre a un nombre de Milnor constant est à type topologique constant, donc équisingulière. Il faut attendre $\left[\mathrm{T}_{5}\right]$ pour que $B$. TEISSIER donne une démonstration algèbrique de ce résultat.

Dans $\left[\mathrm{T}_{2}\right]$ B. TEISSIER démontre l'équivalence de l'équisingularité et d'une condition de résolution simultanée des singularités. 


\section{INTRODUCTION}

En résumé toutes les définitions "raisonnables" d'équisingularité sont équivalentes dans le cas des germes de courbes planes, (citons $\left[\mathrm{T}_{5}\right]$ ou $\left[\mathrm{T}_{2}\right]$ pour des exposés plus détaillés).

DIFFÉRENTES NOTIONS D'ÉQUISINGULARITE POUR LES GERMES DE COURBES GAUCHES.

Par souci de clarté, nous nous sommes volontairement limités à l'étude des familles de courbes paramètrées par un espace lisse de dimension un, bien que tous les résultats que nous exposons semblent se généraliser facilement au cas des familles paramétrées par un espace lisse de dimension quelconque.

Nous travaillons donc dans la situation suivante $: X \subset \mathbb{C} \times \mathbb{C}^{\mathrm{N}}$ est un germe de surface tel que la restriction $p$ à $X$ de la projection canonique de $\mathbb{a} \times \mathbb{a}^{N}$ sur $U=\mathbb{C} \times\{0\}$ soit plate, $x_{0}=p^{-1}(0)$ soit un germe de courbe réduite de $\mathbb{C}^{N}$, et le lieu singulier relatif (à $p$ ) de $X$ soit $U$. On peut alors introduire les différentes notions d'équisingularité suivantes :

(1) TRIVIALITÉ TOPOLOGIQUE : la paire $\left(B_{\varepsilon}, X_{u}\right)$ reste homéomorphe à $\left(B_{\varepsilon}, X_{0}\right)$ au voisinage de $u=0$, lorsque $B_{\varepsilon}$ désigne la boule de $\mathbb{C}^{N}$ de centre 0 et de rayon $\varepsilon$ assez petit, et $x_{u}=p^{-1}(u) \cap B_{\varepsilon}$.

(2) $\mu$ CONSTANT : le nombre de Milnor $\mu\left(X_{u}\right)$ de la fibre $X_{u}$ est constant au voisinage de 0 .

(3) LES CONDITIONS DE WHITNEY : le couple $(X-U, U)$ vérifie les conditions a) et b) de Whitney au voisinage de 0 .

(4) L'ÉQUISATURATION : les algèbres saturées $\tilde{O}_{X_{u}}$ sont isomorphes lorsque $u$ varie dans un voisinage de $0\left(\mathcal{O}_{X_{u}}\right.$ désignant l'algèbre de $X_{u}$ en ce point spécial).

Introduisons encore, suivant J. STUTZ [St] :

(3') $\operatorname{dim} C_{4}(x)=2$ : où $C_{4}(x)$ est le cône ensemble des limites en 0 des vecteurs tangents à la partie lisse de $x$. 


\section{J. BRIANÇON, A. GALLIGO, M. GRANGER}

(4') $\operatorname{dim} C_{4}(x)=2$ et $\operatorname{dim} C_{5}(x)=3$ : où $C_{5}(x)$ est le cône ensemble des limites en 0 des vecteurs sécants à $X$.

Notons aussi $\delta\left(X_{u}\right)=\operatorname{dim} C\left(\overline{\mathcal{O}}_{X_{u}} / \mathcal{O}_{X_{u}}\right)$ (oū $\overline{\mathcal{O}}_{X_{u}}$ est l'algèbre normalisée de $\left.\mathcal{Q}_{X_{u}}\right)$; $r\left(x_{u}\right)$ le nombre de composantes irréductibles du germe $x_{u} ; m\left(X_{u}\right)$ sa multiplicité et, si $X_{u}^{\prime}$ désigne la projection plane générique de $X_{u}, \ell\left(X_{u}\right)=\delta\left(X_{u}^{\prime}\right)-\delta\left(X_{u}\right)$.

Enfin, suivant $B$. TEISSIER $\left[\mathrm{T}_{2}\right]$, nous introduisons aussi :

(2"') résolution simultanée faible.

(3"') résolution simultanée forte.

On a le diagramme suivant d'implications :

(1) trivialité topologique

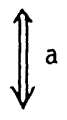

(2"') $)$ résolution simultanée
faible $\stackrel{c}{\Leftrightarrow}(2)$ uconstant $\stackrel{b}{\Leftrightarrow}\left(2^{\prime \prime}\right) \delta\left(X_{u}\right)$ et $\underset{a u}{ } r\left(X_{u}\right)$ constants<smiles>[CH]=C</smiles>
voisinage de 0

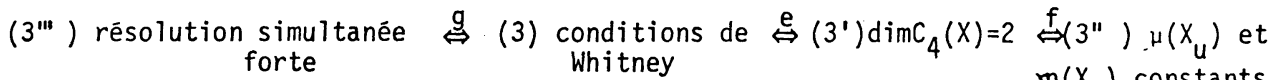

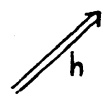
$m\left(X_{u}\right)$ constants au voisinage de 0
(4) équisaturation $\stackrel{i}{\Leftrightarrow}$
$\stackrel{i}{\Leftrightarrow}\left(4^{\prime}\right)$ et $\operatorname{dim}_{5}(X)=3$
$\stackrel{j}{\Leftrightarrow}\left(4^{\prime \prime}\right) \mu\left(X_{u}\right)$ et $\ell\left(X_{u}\right)$ sont constants au voisinage de 0

$a, b$ et $c:$ R.0. BUCHWEISS et G.M. GREUEL

d : THCH MATHER (et a)

e et $i$ : J. STUTZ

$g:$ B. TEISSIER

$f, h, j:$ démontrés ici. 
QUELQUES ÉLÉMENTS DE COMPARAISON.

Dans le chapitre $V$ nous donnons divers exemples illustrant des différences importantes avec l'équisingularité des courbes planes.

Notons que :

1) Les réciproques de $d$ ) et $h$ ) sont toutes les deux fausses :

7d : exemple V.1. de J.P.G. HENRY avec $X_{0}$ intersection complète. exemple V.2. avec $X_{0}$ irréductible, Gorenstein.

Th : exemple V.3. avec $x_{0}$ intersection complète irréductible de semi-groupe $\Gamma\left(X_{u}\right)$ constant.

2) Le critère discriminant de Zariski se généralise au cas des intersections complètes où il équivaut aux conditions de Whitney (théorème III.5 et Remarque III.6). Par contre, nous donnons un exemple (V.5) d'une famille de courbes gauches pour laquelle les conditions de Whitney sont satisfaites mais où la multiplicité du discriminant générique varie.

3) Dans la déformation semi-universelle d'un germe de courbe plane la strate d'équisingularité est lisse. Ce résultat est du à J. WAHL qui utilise la définition de l'équisingularité à l'aide de la (a)-équivalence ([Wa ]). Dans le cas des germes de courbes irréductibles une autre démonstration est donnée par $B$. TEISSIER dans $\left[\mathrm{T}_{3}\right]$ qui déduit ce résultat de la lissité de la "strate à semi groupe constant".

Nous donnons un exemple de germe de courbe gauche $(V, 6)$ dont la déformation semi-universelle a une "strate à $\mu$ constant" singulière.

4) Dans le cas des germes de courbes planes les notions d'équivalence et d'équisingularité sont liées par la propriété suivante: Etant donnés deux germes de courbes planes $C$ et $D$ topologiquement équivalents, il existe une dēformation à un paramètre équisingulière contenant deux fibres isomorphes respectivement à $C$ et $D$. 


\section{J. BRIANÇON, A. GALLIGO, M. GRANGER}

Dans le cas des germes de courbes gauches nous ne disposons pas d'une définition satisfaisante de l'équivalence de deux germes.

Ainsi deux germes de courbes dans $\mathbf{C}^{3}$ sont topologiquement équivalents si et seulement si ils ont le même nombre de branches. Cela ne peut suffire en général pour les joindre par une famille équisingulière en aucun des sens étudiés ici. 5) Signalons qu'à notre connaissance la question suivante n'est pas résolue : une déformation plate à $\mu$ constant d'un germe de courbe irréductible intersection complète est elle à semi-groupe constant ? ou au moins équimultiple ? Pour terminer cette introduction attirons l'attention du lecteur sur deux points particuliers qui nous semblent originaux :

D'une part le fait que le nombre de composantes globales dans la boule $B_{\varepsilon}$ de la fibre générique $x_{u}$ d'une déformation plate de la courbe $X_{0}$ soit égal au nombre de composantes du germe de surface $X$ (théorème $I, 9$ ).

D'autre part l'étude de l'invariant analytique $l\left(X_{0}\right)$ mesurant la différence entre la courbe $x_{0}$ et sa projection plane générique ainsi que la description des directions de projections planes non génériques de $x_{0}$.

Précisément nous interprétons $\ell\left(X_{0}\right)=\delta\left(X_{0}^{1}\right)-\delta\left(X_{0}\right)$ comme le nombre de points doubles de la projection plane générique d'une déformation lisse de $x_{0}$ et nous montrons que $\ell\left(X_{u}\right)$ est semi-continu (proposition IV. 6 et corollaire IV.7). Nous montrons que l'ensemble des 1 imites de vecteurs sécants à $x_{0}$ est la réunion d'un nombre fini de 2-plans (proposition IV.1) ; la projection plane $\pi_{H}$ parallèlement à un $N-2$ plan $H$ de la courbe $x_{0}$ est non générique si et seulement si $H$ contient $l^{\prime}$ 'un non nul de ces vecteurs, ce qui équivaut au fait que :

$$
\delta\left(\pi_{H}\left(X_{0}\right)\right)-\delta\left(X_{0}\right)>\ell\left(X_{0}\right)
$$

ou éventuellement $\pi_{H}\left(x_{0}\right)$ non réduit. 


\section{INTRODUCTION}

La matière de l'appendice provient en grande partie d'un manuscrit non publié de F. PHAMet B. TEISSIER décrivant la construction du saturé lipschitzien d'une algèbre analytique locale de dimension un (qui coïncide ici avec le saturé de Zariski) à partir de la suite de ses "exposants caractéristiques" dans le cas irréductible (théorème VI.1.6.) et dans le cas réductible que nous avons précisé (théorème VI.2.2., Remarque et lemme VI.3.6) • Remarquons que ces calculs sont analogues à certains calculs de 0 . ZARISKI dans $\left[\mathrm{Z}_{4}\right]$.

Nous utilisons cette description pour montrer que deux courbes ont même saturé si et seulement si leurs projections planes génēriques ont même type topologique (théorème VI.0.2). C'est ce résultat qui nous permet justement au chapitre IV de définir la projection plane générique à équivalence topologique près et de montrer que l'équisaturation équivaut à l'équisingularité de la projection plane générique. 


\section{P $\quad$ L $\quad A \quad N$}

I. Dēformations plates d'un ģerme de courbe réduite.

II. " $\mu$ constant ".

III. Conditions de Whitney.

IV. Equisaturation.

V. Exemples.

VI. Appendice : Saturé des algèbres analytiques locales de dimension 1 d'après F. PHAM et B. TEISSIER. 


\section{ChaP, I : DEFORMATIONS PLATES D'UN GERME DE COURBE RÉ⿴囗十ITE.}

Soit $x_{0}$ un germe de courbe réduite de $\left(\mathbb{C}^{N}, 0\right)$ défini par l'idéal $I_{0}=\left(f_{1}, \ldots, f_{k}\right) \subset \mathbb{Q}\left\{x_{1}, \ldots, x_{N}\right\}$ et soit $X$ un germe de surface de $\left(\mathbb{C}^{N+1}, 0\right)$ défini par l'idéal I de $\mathbb{C}\left\{u, x_{1}, \ldots, x_{N}\right\}$ engendré par les éléments $\left(F_{1}, \ldots, F_{k}\right)$ avec, pour $i=1, \ldots, k, F_{i}\left(0, x_{1}, \ldots, x_{N}\right)=f_{j}\left(x_{1}, \ldots, x_{N}\right)$. Notons $\mathrm{p}$ la restriction à $X$ de la projection canonique de $\left(\mathbb{C}^{N+1}, 0\right)$ sur le premier facteur $(\mathbb{C}, 0)$; lorsque $p$ est plate, c'est à dire lorsque $\mathcal{O}_{X}=\frac{\mathbb{C}\left\{u, x_{1}, \ldots, x_{N}\right\}}{I}$ est un $\mathfrak{Q}\{u\}$-module plat, nous dirons que $X$ est une déformation plate à un paramètre de $x_{0}$.

LEMME 1.1.: Si $X$ est une déformation à un paramètre de la courbe réduite $X_{0}$, les conditions suivantes sont équivalentes :

(i) $X$ est une déformation plate

(ii) $X$ est de Cohen- Macaulay

(iii) $X$ est de dimension pure 2 .

Preuve :

(i) $\Rightarrow$ (ii) : u est non diviseur de 0 dans $\mathbb{Q}_{x}$, et, puisque $x_{0}$ est réduite, il existe un paramètre, $x_{1}$ par exemple, non diviseur de 0 dans $C_{x_{0}}=Q_{X} / u Q_{x}$. La suite $\left(u, x_{1}\right)$ est donc régulière dans $\mathcal{O}_{x}$.

(ii) $\Rightarrow$ (iii) : est évident.

$(i i i) \Rightarrow(i): \operatorname{dim}\left(\mathcal{O}_{X} / u \mathcal{O}_{X}\right)=\operatorname{dim} \mathcal{O}_{X_{0}}=\operatorname{dim} \mathcal{O}_{X}-1$, et, dans $\mathcal{O}_{X}$ de dimension 
pure, cela implique que $u$ est non diviseur de 0 et donc que $\mathcal{O}_{X}$ est un $\mathbb{C}\{u\}$-module plat?

\section{LEMME I.2.}

Soient $X$ une déformation plate à un paramètre de la courbe réduite $x_{0}$ et $x^{1}, \ldots, x^{r}$ les composantes irréductibles de $x$. La restriction de $p$ à toute union $X^{1} \cup . . U X^{i}(1 \leq i \leq r)$ de composantes irréductibles est plate.

PREUVE :

D'après le lemme I.1., X est de dimension pure 2, donc il en est de même pour $x^{1} \cup \ldots U X^{i}$ et on conclut de la même manière que pour (iii) $\Rightarrow(i)$ dans le Temme précédenta

REMARQUE I.3.:

$X^{1}$, par exemple, est donc une déformation plate de sa fibre $\left(X^{1}\right)_{0}$ : mais $\left(X^{1}\right)_{0}$ $n$ 'est pas en général une courbe réduite et peut avoir une composante immergée à l'origine. Voir l'exemple V.1.

LEMME I.4.:

Avec les hypothèses du lemme I.2., la dimension de $\left(X^{1} \cup X^{2} \cup \ldots \cup X^{i}\right) \cap\left(X^{i+1} \cup \ldots \cup X^{r}\right)$ est $1 .(1 \leq i<r)$.

PREUVE :

I1 s'agit de montrer que 1'intersection $\left(X^{1} \cup \ldots \cup X^{i}\right) \cap\left(X^{i+1} \cup \ldots U X^{r}\right) n^{\prime}$ est pas contenu dans $\{0\} \times \mathbb{C}^{N}$, supposons le contraire : en désignant par $I^{1}, I^{2}, \ldots, I^{r}$ les idéaux premiers définissant respectivement $x^{1}, x^{2}, \ldots, x^{r}$, nous aurions :

$$
u^{\alpha} \in\left(I^{1} \cap \ldots \cap I^{i}\right)+\left(I^{i+1} \cap \ldots \cap I^{r}\right)
$$

supposons $\alpha \in \mathbb{N}$ choisi le plus petit possible, $u^{\alpha}=g_{1}-h_{1}$ avec $g_{1} \in\left(I^{1} \cap \ldots \cap I^{i}\right)$ et $h_{1} \in\left(I^{i+1} \cap \ldots \cap I^{r}\right)$. On a donc :

$g_{1}\left(0, x_{1}, \ldots, x_{N}\right)=h_{1}\left(0, x_{1}, \ldots, x_{N}\right) \in\left(I^{1} \cap \ldots \cap I^{i}\right)_{0} \cap\left(I^{i+1} \cap \ldots \cap I^{r}\right)_{0}$ 


\section{DÉFORMATIONS ÉQUISINGULIÈRES}

$\left(I^{1} \cap \ldots \cap I^{i}\right)_{0}$ et $\left(I^{i+1} \cap \ldots \cap I^{r}\right)_{0}$ désignant les idéaux de $\mathbb{a}\left\{x_{1}, \ldots, x_{N}\right\}$ définissant les fibres $\left(X^{1} \cup \ldots \cup X^{i}\right)_{0}$ et $\left(X^{i+1} \cup \ldots \cup X^{r}\right)_{0}$; mais on a $I_{0} \subset\left(I^{1} \cap \ldots \cap I^{i}\right)_{0} \cap\left(I^{i+1} \cap \ldots \cap I^{r}\right)_{0}$; $I_{0}$ étant réduit, grâce à l'égalité ensembliste $\left|x_{0}\right|=\left|\left(x^{1} \cup \ldots \cup x^{i}\right)_{0} \cup\left(x^{i+1} \cup \ldots \cup x^{r}\right)_{0}\right|$ on déduit l'égalité des idéaux $I_{0}=\left(I^{1} \cap \ldots \cap I^{i}\right)_{0} \cap\left(I^{i+1} \cap \ldots \cap I^{r}\right)_{0}$ et donc $I^{\prime}$ 'existence d'un élément $g\left(u, x_{1}, \ldots, x_{N}\right) \in I$ vérifiant : $g\left(0, x_{1}, \ldots, x_{N}\right)=g_{1}\left(0, x_{1}, \ldots, x_{N}\right)=h_{1}\left(0, x_{1}, \ldots, x_{N}\right)$. Ecrivons alors : $u^{\alpha}=\left(g_{1}-g\right)-\left(h_{1}-g\right), g_{1}-g=u g_{1}^{\prime}, h_{1}-g=u h_{1}^{\prime}$ d'après le lemme I.2, $g_{1}^{i} \in\left(I^{1} \cap \ldots \cap I^{i}\right)$ et $h_{1}^{i} \in\left(I^{i+1} \cap \ldots \cap I^{r}\right)$; comme $u^{\alpha-1}=g_{1}^{\prime}-h_{1}^{\prime}$ ceci contredit $l^{\prime}$ hypothèse faite sur a d'être minimum DÉFINITION I.5. :

On dit que la déformation $x$ de $x_{0}$ est centrée si le lieu singulier de $x$, Sing $(X)$, a pour ensemble sous-jacent $\mathbb{C}\{0\}$. On dit qu'une composante irréductible $X^{1}$ de $X$ est essentielle si $X^{1}$ contient $\mathbb{C} \times\{0\}$. PROPOSITION I.6. :

Si $X$ est une déformation plate centrée de $X_{0}$, toutes les composantes irréductibles de $X$ sont essentielles.

\section{PREUVE :}

Si $x$ est irréductible, il n'y a rien à montrer ; sinon, $x^{1} \cap\left(X^{2} U \ldots \cup X^{r}\right)$ est contenu dans le lieu singulier de $X$, et d'après le lemme précédent, n'est pas contenu dans $\{u=0\} ; c^{\prime}$ est donc que $x^{1}$ contient $\mathbb{C} \times\{0\}$ Reprenons maintenant le cas général où $X$ est une déformation plate à un paramètre de la courbe réduite $X_{0}$; nous désignons par la même lettre un germe et son 
représentant "assez petit".

Précisément soient $U=\{u \in \mathbb{C} /|u|<\eta\}, B_{\varepsilon}=\left\{x \in \mathbb{C}^{\mathbb{N}} /\left|x_{1}\right|^{2}+\ldots+\left|x_{N}\right|^{2}<\varepsilon^{2}\right\}$ $S_{\varepsilon^{\prime}}=\left\{x \in \mathbb{C}^{N} /\left|x_{1}\right|^{2}+\ldots+\left|x_{N}\right|^{2}=\varepsilon^{\prime 2}\right\}$ tels que :

a) 0 est le seul point singulier de $X_{0}$ dans la boule fermée $\bar{B}_{\varepsilon}, X_{0}$ est transverse à la sphère $S_{\varepsilon}$ ! pour $0<\varepsilon^{\prime} \leq \varepsilon-i l$ en résulte que. la paire $\left(X_{0} \cap \bar{B}_{\varepsilon}, \bar{B}_{\varepsilon}\right)$ est homèomorphe $\bar{a}\left(C_{0}, \bar{B}_{\varepsilon}\right), C_{0}$ désignant le cône de sommet 0 sur $X_{0} \cap S_{\varepsilon}$ ( $[M i]$, th.2.10); de plus on sait que $x_{0} \cap S_{\varepsilon}$ est difféomorphe à l'union de $r_{0}$ cercles disjoints, $r_{0}$ étant égal au nombre de composantes irréductibles de $x_{0}-$

b) le lieu singulier et le lieu singulier relatif (à $p$ ) de $X$ coincident dans $U \times \bar{B}_{\varepsilon}\left(\left[T_{1}\right]\right.$ th. 2.8), et $X$ reste transverse à $U \times S_{\varepsilon}$.

c) Sing $X-\{0\}$ est un revêtement de $U-\{0\}$ (Sing $(X)$ désignant le lieu singulier réduit). Enfin la paire $\left(X_{1 \text { isse }}\right.$, Sing $\left.(X)\right)$ vérifie les conditions de Whitney dans $(U-\{0\}) \times B_{\varepsilon}([W]$ lemme 19.3):

Nous savons alors, par le théorème de Thom-Mather ( $\left[\mathrm{Ma}_{1}\right]$ Prop 11.1) que pour deux points $u_{1}$ et $u_{2}$ de $U-\{0\}$ les fibres $x_{u_{1}}$ et $X_{u_{2}}$ de $p$ dans $B_{\varepsilon}$ sont homéomorphes. Nous pouvons donc parler du type topologique de la fibre générique $x_{u}$ (ou de la triade $\left(\operatorname{Sing}\left(X_{u}\right), X_{u}, B_{\varepsilon}\right)$ ). En particulier le nombre $\tilde{r}$ de composantes irréductibles de la fibre générique $x_{u}$ est bien défini, puisque $c^{\prime}$ est le nombre de composantes connexes de $X_{u}-\operatorname{sing}\left(x_{u}\right)$. Pour tout lacet $\gamma$ d'origine $u_{1}$ dans $U$ - \{(j\}, le premier lemme d'isotopie de Thom fournit également un homéomorphisme "de monodromie" $\gamma^{*}$ de $x_{u_{1}}$ dans lui même, qui donne une permutation des composantes irréductibles de $x_{u_{1}}$.

Enfin remarquons que tout ceci est bien défini dẽs que $X$ est de dimension pure 2 et $x_{0}$ à singularité isolée.

Nous allons maintenant comparer le nombre $r$ de composantes irréductibles de $X$ et le nombre $\tilde{r}$ de composantes irréductibles de la fibre générique $X_{u}$ et nous appercevoir qu'en fait $r=\tilde{r}$ ! 
LEMME I.7. Soit $G(u, x, y)$ un germe de fonction analytique à l'origine de $c^{3}$ définissant un germe $Y$ de sous espace analytique irréductible ; si $g(x, y)=G(0, x, y)$ n'est pas la puissance (d'ordre au moins égale à 2) d'une fonction analytique, la fibre générique $Y_{u}$ est irréductible.

PREUVE :

grâce au théorème de préparation de Weierstrass, nous pouvons nous placer dans les conditions suivantes : $G(u, x, y)=y^{p}+a_{1}(u, x) y^{p-1}+\ldots+a_{p}(u, x)$ où $a_{1}, \ldots, a_{p}$ sont des fonctions analytiques dans le polydisque $U \times \mathbb{D}_{\varepsilon_{1}}\left(\mathbb{D}_{\varepsilon_{1}}=\left\{x \in \mathbb{C} /|x|<\varepsilon_{1}\right\}\right)$ nulles à l'origine; les $\mathrm{p}$ racines du polynome normalisé $G$ sont dans le disque $D_{\varepsilon_{2}}=\left\{y \in \mathbb{C} /|y|<\varepsilon_{2}\right\}$ et $Y$ est défini par $G$ dans $U \times D_{\varepsilon_{1}} \times D_{\varepsilon_{2}}$. Le discriminant $\Delta$ du polynome $G$ est :

$\Delta=B u^{\alpha}\left[x^{\sigma}+a_{1}(u) x^{\sigma-1}+\ldots+a_{\sigma}(u)\right]$.

où $\beta$ est une unité de $\left.\mathcal{Q} U \times D_{\varepsilon_{1}}\right), a_{1}(u) \ldots a_{\sigma}(u)$ sont des fonctions analytiques dans $U$, nulles en 0 ; de plus nous supposons que la restriction à $U-\{0\} d u$ lieu discriminant réduit est un revêtemient de $U-\{0\}$.

Le critère discriminant de Zariski $\left(\left[Z_{1}\right]\right.$ th. 7), puis le théorème de Thom Mather nous permettent alors d'affirmer que, localement dans $U-\{0\}$, la famille de courbes planes $Y_{u}$ est équisingulière, donc localément topologiquement triviale ; et même mieux, la paire $\left(Y_{u}\right.$, Sing $\left.\left(Y_{u}\right)\right)$ est localement topologiquement triviale. Notons $U^{+}=U-\left[0, n\left[\right.\right.$ (resp. $\left.\left.U^{-}=U-\right]-n, 0\right]$ ) l'ouvert contractile des éléments de $U$ non réels positifs (resp non réels négatifs); $Y_{\mid U^{+}}$(resp $Y_{\mid U^{-}}$) est une fibration topologique triviale et admet donc $\tilde{r}$ composantes irréductibles. Nous pouvons considérer le monoĩde $\mathcal{O}^{0}\left(U^{+}\right)[y]$ des polynomes normalisés comme factoriel ( $[G-F]$ th. III.6.5) la décomposition unique de $G$ correspondant à la décomposition de $\mathrm{Y}_{\mathrm{U}^{+}}$en ses composantes irréductibles : 


$$
G=G_{1}^{+} \times G_{2}^{+} \ldots \times G_{\tilde{r}}^{+} \text {dans } U^{+} \times D_{\varepsilon_{1}} \times D_{\varepsilon_{2}}
$$

De même nous avons $G=G_{1}^{-} \times G_{2}^{-} \ldots \times G_{\tilde{r}}$ dans $U^{-} \times D_{\varepsilon_{1}} \times D_{\varepsilon_{2}}$; et, quitte à renuméroter, nous pouvons supposer

pour $i=1, \ldots, \tilde{r}, G_{i}^{+}=G_{i}^{-}$dans $\{u \in U / \operatorname{Im}(u)<0\}$.

L'unicité de la décomposition permet alors de définir une permutation $\tau$ de $\{1,2, \ldots, \tilde{r}\}$ en posant $G_{i}^{-}=G_{\tau(i)}^{+}$dans $\{u \in U / \operatorname{Im}(u)>0\}$, ceci pour $i=1, \ldots, \tilde{r}$.

a) Montrons d'abord que $\tau n^{\prime}$ admet pas de cycle strict :

supposons, pour fixer les idées, que $\tau$ laisse stable la partie $\{1,2, \ldots, l\}$ avec $\ell<\tilde{r}$; alors les fonctions analytiques $G_{1}^{+} \times G_{2}^{+} \ldots \times G_{l}^{+}$et $G_{1}^{-} \times G_{2}^{-} \ldots \times G_{l}^{-}$ coïncident sur $\left(U^{+} \cap U^{-}\right) \times \mathbb{D}_{\varepsilon_{1}} \times \mathbb{D}_{\varepsilon_{2}}$ et définissent donc une fonction analytique $H$ dans $(U-\{0\}) \times \mathbb{D}_{\varepsilon_{1}} \times \mathbb{D}_{\varepsilon_{2}} ; H$ est bornée au voisinage de $\{0\} \times D_{\varepsilon_{1}} \times D_{\varepsilon_{2}}$ (puisque les coefficients du polynome normalisé H s'obtiennent à partir des fonctions symétriques élémentaires de certaines racines de $G$ ) et se prolonge donc à $U \times \mathbb{D}_{\varepsilon_{1}} \times \mathbb{D}_{\varepsilon_{2}}$; enfin $H$ est un diviseur strict de $G$, ce qui est contraire à 1 'hypothèse "G irréductible".

b) Quitte à renuméroter, nous pouvons supposer que $\tau$ est la permutation circulaire $\tau(i)=i+1$ pour $i=1, \ldots, \tilde{r}-1$ et $\tau(\tilde{r})=1$. Définissons une fonction analytique $H(v, x, y)$ dans $(\mathbb{D}-\{0\}) \times \mathbb{D}_{\varepsilon_{1}} \times \mathbb{D}_{\varepsilon_{\tilde{q}}}$ (où $\mathbb{D}$ est le disque unité) en posant pour $k$ variant de 1 à $\tilde{r}$ :

$$
\left\{\begin{array}{l}
H(v, x, y)=G_{k}^{+}\left(n v^{\tilde{r}}, x, y\right) \text { pour }(k-1) \frac{2 \pi}{\tilde{r}}<\arg v<(k) \frac{2 \pi}{\tilde{r}} \\
H(v, x, y)=G_{k}^{-}\left(n v^{\tilde{r}}, x, y\right) \text { pour }(2 k-1) \frac{\pi}{\tilde{r}}<\arg v<(2 k+1) \frac{\pi}{\tilde{r}}
\end{array}\right.
$$

Comme précédemment $H$ se prolonge à $B \times D_{\varepsilon_{1}} \times D_{\varepsilon_{2}}$ et on obtient, pour tout $k=1 \ldots \tilde{r}, \lim _{\substack{u \rightarrow 0 \\ u \in U^{+}}} G_{k}^{+}(u, x, y)=H(0, x, y)$.

D'où $g(x, y)=[H(0, x, y)]^{\tilde{r}}$ et donc, par hypothèse $\tilde{r}=1$. 


\section{DÉFORMATIONS ÉQUISINGULIÈRES}

LEMME I.8. :

Soit $x^{1}$ une surface de dimension pure dans $x \mathbb{C}^{\mathbb{N}}$ déformation plate à un paramètre d'une courbe à singularité isolée $x_{0}^{1} \subset \mathbb{C}^{N}$. Si $x^{1}$ est irréductible, la fibre générique $x_{u}^{1}$ est irréductible.

PREUVE :

Soit $x$ un paramètre transverse de la courbe réduite $\left|x_{0}^{1}\right|$. Pour presque tout choix de $\lambda=\left(\lambda_{1}, \ldots, \lambda_{N}\right) \in \mathbb{C}^{N}-\{0\}, y=\lambda_{1} x_{1}+\ldots+\lambda_{N} x_{N}$ a pour image dans $\mathcal{O}_{x}=\mathbb{a}\left\{x_{1}, \ldots x_{N}\right\} / I$ (de corps des fractions $L$ ), un élément primitif $\bar{y}$ de l'extension finie $\mathbb{a}((u, x)) \subset L$, et $\bar{y}$ admet un polynome minimal de la forme :

$G=y^{m}+a_{1}(u, x) y^{m-1}+\ldots+a_{m}(u, x), a_{i} \in \mathbb{C}\{u, x\}$.

On note $p: \mathbb{C}^{3} \rightarrow \mathbb{C}^{2}$ et $\pi: \mathbb{C} \times \mathbb{C}^{\mathbb{N}} \rightarrow \mathbb{C} \times \mathbb{C}^{2}$ les projections $p(u, x, y)=(u, x)$, $\pi\left(u, x_{1}, \ldots, x_{N}\right)=(u, x, y), Y=G^{-1}(0), \delta(u, x)=\operatorname{Res}\left(G, G_{y}^{\prime}\right)$ le discriminant de $p_{\mid Y}$ et $\Delta=p^{-1}\left(\delta^{-1}(0)\right)$. Alors d'après $[N$, lemme 4 p.37], on a un isomorphisme $\pi: X^{1}-\pi^{-1}(\Delta) \stackrel{\gtrless}{\rightarrow} Y-\Delta$. On peut également supposer (cf ch IV) que $\pi$ induit un isomorphisme $X_{0}^{1}-\{0\} \rightarrow \pi\left(X_{0}^{1}\right)-\{0\}$. On remarque que $Y=\pi\left(X^{1}\right)$ et que l'idéal In $\mathbb{C}\{u, x, y\}$ qui dēfinit ensemblistement $\pi\left(X^{1}\right)$ est premier, donc égal $(G)$. Ainsi pour $u \neq 0, \pi: x_{u}^{1} \rightarrow Y_{u}$ est un isomorphisme sauf eventuellement en un nombre fini de points et le lemme résultera de l'irréductibilité de $Y_{u}$. Comme $Y$ est de dimension pure donc déformation plate de sa fibre $Y_{0}$, l'irréductibilité de $Y_{u}$ est assurée d'après le lemme I.7 si $g(x, y)=G(0, x, y)$ n'est pas une puissance. D'après 1 'hypothèse pour tout $\left(x_{0}, y_{0}\right) \in \pi\left(x_{0}^{1}\right)-\{0\}, \pi^{-1}\left(x_{0}, y_{0}\right) \cap x_{0}^{1}$ est réduit à un point $\left(x_{0}, y_{0}, z_{0}\right)$ au voisinage duquel $x^{1}$ est une déformation plate d'une courbe lisse réduite. Il en résulte que $Y_{0}$ est lisse en $\left(x_{0}, y_{0}\right)$, donc que $g(x, y)$ est réduit en ce point.

Des lemmes I.7 et 1.8 on déduit: 
THÉOREME I.9. :

Soient $X$ une déformation plate à un paramètre de la courbe réduite $X_{0}$ et $x^{1}, \ldots, x^{r}$ les composantes irréductibles de $x$. Les composantes irréductibles de la fibre générique $X_{u}$ sont les fibres génériques $X_{u}^{1}, \ldots, X_{u}^{r}$ des composantes irréductibles de $x$.

COROLLAIRE I.10. :

Soit $X$ une déformation plate, à un paramẽtre de la courbe réduite $X_{0}$; la fibre générique $X_{u}$ est connexe.

PREUVE. :

Chaque composante irréductible $x_{u}^{i}$ étant connexe les composantes connexes de $X_{u}$ sont des réunions des $X_{u}^{i}$. D'après le lemme $I .4, X_{u}$ est donc connexe.

REMARQUE I.11. :

La connexité de $X_{u}$ est démontrée dans ( $\left.[B] \bar{G}\right]$, 3.2) de manière tout à fait différente.

REMARQUE 1.12. : Comme cas particulier du corollaire I.10 noc's dbtenons: Si $x_{0}$ est une intersection complète $f_{1}=\ldots=f_{N-1}=0$ la courbe de niveau générique $f_{1}-u_{1}=\ldots=f_{N-1}-u_{N-1}=0$ est lisse et connexe. 


\section{CHAP, II : "}

Dans ce chapitre nous allons prendre comme critère d'équisingularité la constance du nombre $\mu$ de Milnor. Nous rappellerons des résultats de B. Teissier et de R.0. Buchweiss et G.M. Greuel, dans le cas d'une deformation plate centrée. DÉFINITION II.1. Soit $x_{0}$ un germe de courbe réduite plongé dans $\mathbb{C}^{N}$, d'anneau local $\mathcal{O}_{x_{0}}$; on désignera par :

- $n^{*}: \mathcal{O}_{x_{0}} \hookrightarrow \overline{\mathcal{O}}_{x_{0}}$ le morphisme de normalisation

$-m\left(x_{0}\right)$ la multiplicité de $x_{0}$

$-r\left(x_{0}\right)$ le nombre de composantes irréductibles de $x_{0}$

$-\delta\left(X_{0}\right)$ le nombre entier $\delta\left(X_{0}\right)=\operatorname{dim}_{\mathbb{C}}\left(\bar{O}_{X_{0}} / \mathcal{O}_{X_{0}}\right)$

- $\mu\left(X_{0}\right)$ le nombre de Milnor dont la définition généralisée a été donnée par R.0. Buchweiss et G.M. Greuel dans [B-G] :

$$
\mu\left(X_{0}\right)=\operatorname{dim}_{\mathbb{C}}\left(\omega_{X_{0}} / \operatorname{Im} d\right)
$$

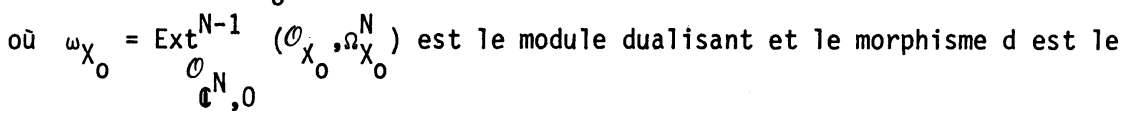
composé des morphismes suivants :

$$
\underset{x_{0}}{0} \rightarrow \Omega_{x_{0}} \rightarrow n_{*} \Omega \bar{x}_{0} \simeq n_{*}{ }^{\omega} \bar{x}_{0} \rightarrow{ }^{\omega} x_{0}
$$

la première flèche étant la dérivation extérieure.

Buchweiss et Greuel montrent la formule :

$$
\mu\left(X_{0}\right)=2 \delta\left(X_{0}\right)-r\left(X_{0}\right)+1
$$

démontrée dans le cas des courbes planes par Milnor ([Mi] th. 10.5) et dans le cas des intersections complètes par Giusti ( $\left.\mathrm{Gi}_{2}\right]$ App. 1). 
DÉFINITION II.2. :

Soit $p: X \rightarrow U \subset \mathbb{Q}$ une déformation plate centrée d'un germe de courbe réduite $x_{0}$. On dira que $p$ admet une résolution simultanée très faible si le morphisme de normalisation $n: \bar{X} \rightarrow X$ vérifie :

TF 1) $\bar{X}$ est non singulier et $p \circ n$ est une submersion analytique.

TF 2) pour tout $u \in U$ le morphisme induit $(\bar{X})_{u} \rightarrow \dot{x}_{u}$ est la normalisation. On dira que $p$ admet une résolution faible $n: \bar{X} \rightarrow X$ si $n$ vérifie TF 1), TF 2) et :

FA) le morphisme induit par restriction $p \circ n:\left(n^{-1}(U)\right)_{\text {red }} \rightarrow U$ est simple, donc étale.

REMARQUE. :

Si $\mathrm{p}$ admet une résolution simultanée très faible nous dirons aussi que $X$ admet une paramètrisation en famille, c'est à dire que $X$ est $l$ 'image réduite du morphisme

$$
\begin{gathered}
n:(\mathbb{a}, 0) \times \underset{j=1}{\Perp}\left(\mathbb{G}_{j}, 0\right) \rightarrow\left(\mathbb{C} \times \mathbb{C}^{N}, 0\right) \\
\text { défini par N éléments } z_{i}=z_{j}\left(u, t_{j}\right) \in \prod_{j=1}^{r} \mathbf{r}\left\{u, t_{j}\right\}(1 \leq i \leq N): \\
n\left(u, t_{j}\right)=\left(u, z_{1}\left(u, t_{j}\right), \ldots, z_{N}\left(u, t_{j}\right)\right)
\end{gathered}
$$

et oũ le morphisme induit pour $u=0$ est la normalisation de $x_{0}$.

THÉORËME II.3. : (B. Teissier : $\left[\mathrm{T}_{2}\right]$ th. 1 et $\left[\mathrm{T}_{1}\right]$ p. 61).

Soit $p: X \rightarrow U \subset \mathbb{C}$ une déformation plate centrée d'un germe de courbe réduite $X_{0}$ et soit $n: \bar{x} \rightarrow X$ la normalisation alors :

1) $P$ on est plat et $\delta\left((\bar{X})_{0}\right)=\delta\left(X_{0}\right)-\delta\left(X_{u}\right)$ pour tout $u \in U \backslash\{0\}$ assez petit.

2) les conditions suivantes sont équivalentes :

i) p admet une résolution simultanée très faible

ii) $\delta\left(X_{u}\right)=\delta\left(X_{0}\right)$ pour tout $u \in U$ assez petit. 


\section{DÉFORMATIONS ÉQUISINGULIÈRES}

THËORÈME II.4. : (R.0. Buchweiss et G.M. Greuel : [B.G] 3.2 et 4.1)

Soit $p: X \rightarrow U \subset \mathbb{C}$ une déformation plate centrée d'un germe de courbe réduite $X_{0}$. Notons $X_{u}$ la fibre générique, $u\left(X_{u}\right), \delta\left(X_{u}\right)$ et $r\left(X_{u}\right)$ ses invariants au point $u \in U$. Alors si $U$ est assez petit

1) $\mu\left(X_{0}\right)-\mu\left(X_{u}\right)=\operatorname{dim}_{\mathbb{C}} H^{1}\left(X_{u}, \mathbf{C}\right)$

et $\mu\left(X_{0}\right)-\mu\left(X_{u}\right) \geq \delta\left(X_{0}\right)-\delta\left(X_{u}\right)$

pour tout $u \in U \backslash\{0\}$

2) les conditions suivantes sont équivalentes :

i) $\mu\left(X_{u}\right)$ est constant pour $u \in U$

ii) $\delta\left(X_{u}\right)$ et $r\left(X_{u}\right)$ sont constants

iii) p admet une résolution simultanée faible

iv) il existe un homéomorphisme de paire entre $\left(B_{\varepsilon}, X_{0}\right)$ et $\left(B_{\varepsilon}, X_{u}\right)$, pour tout $u \in U$.

L'invariant $\delta$ d'un germe de courbe irréductible se calcule à l'aide du semigroupe des valuations de l'anneau local. Dans le cas général nous avons la proposition suivante :

PROPOSITION II.5. :

Soit $x_{0}$ un germe de courbe réduite de $c^{N}$ et soient $c^{1}, \ldots, c^{r}$ ses composantes irréductibles alors:

$\delta\left(x_{0}\right)=\sum_{j=1}^{r} \delta\left(c^{j}\right)+\sum_{j=1}^{r-1}\left(c^{j} ; c^{j+1} u \ldots u c^{r}\right)$

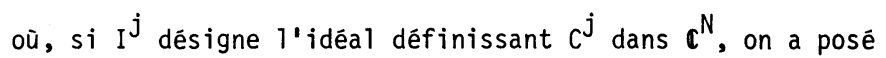

$\left(C^{j} ; C^{j+1} u \ldots v C^{r}\right)=$ colongueur $\left(I^{j}+\left(I^{j+1} \cap \ldots \cap I^{r}\right)\right)$.

\section{PREUVE :}

I1 suffit de démontrer que pour deux germes de courbes réduites $c^{1}$ et $c^{2}$ définies par $\mathrm{I}^{1}$ et $\mathrm{I}^{2}$ on $\mathrm{a}$ : 
J. BRIANÇON, A. GALLIGO, M. GRANGER

$\delta\left(C^{1} \cup C^{2}\right)=\delta\left(C^{1}\right)+\delta\left(C^{2}\right)+$ colongueur $\left(I^{1}+I^{2}\right)$.

Posons $O_{C^{1}}=O_{C^{N}, 0} / I^{1}, O_{C^{2}}=O_{\mathbb{C}^{N}, 0} / I^{2}, O_{C^{1} U C^{2}}=O_{C^{N}, 0} / I^{1} \cap I^{2}$;

de la suite exacte :

$$
\begin{aligned}
& 0 \rightarrow \mathbb{C}_{\mathbb{C}^{N}, 0} / I^{1} \cap I^{2} \stackrel{\alpha}{\rightarrow} \mathcal{O}_{\mathbb{C}^{N}, 0} / I^{1} \oplus \mathcal{O}_{\mathbb{C}^{N}, 0} / I^{2} \stackrel{\beta}{\rightarrow}{\underset{\mathbb{C}}{N}, 0}^{N} / I^{1}+I^{2} \rightarrow 0 \\
& \alpha(h)=(h, h) \text { et } \beta(h, k)=h-k,
\end{aligned}
$$

et des inclusions :

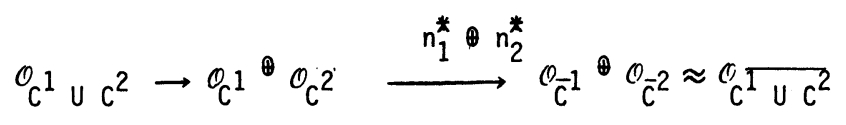

on déduit que :

$$
\begin{aligned}
& \delta\left(c^{1} \cup c^{2}\right)=\operatorname{dim}_{c}{ }_{c^{1} \cup c^{2}} / \mathscr{O}_{c^{1} \cup c^{2}}
\end{aligned}
$$

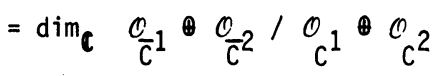

$$
\begin{aligned}
& +\operatorname{dim}_{C}{ }_{C^{1}}^{O} \otimes O_{C^{2}} /{ }_{C^{1}}^{O_{1}} \cup c^{2} \\
& =\delta\left(C^{1}\right)+\delta\left(C^{2}\right)+\operatorname{dim}_{c}{ }_{C^{N}, 0}^{O} /\left(I^{1}+I^{2}\right) .
\end{aligned}
$$

REMARQUE II.6. :

$\left(C^{1} ; C^{2}\right)=$ colongueur $\left(I^{1}+I^{2}\right)$ est une façon naîve de définir la multiplicité $d^{\prime}$ intersection des germes $C^{1}$ et $C^{2}$ définis par les idéaux $I^{1}$ et $I^{2}$ dans $C^{N}$. Cette définition a, entre autre, 1 'inconvénient suivant : si $c^{1}, c^{2}, c^{3}$ sont trois germes de courbes $\left(c^{1} ; c^{2} \cup c^{3}\right)$ n'est en général ni inférieur ni supérieur à $\left(c^{1} ; c^{2}\right)+\left(c^{1} ; c^{3}\right)$.

Voici deux exemples:

20 


$$
\begin{aligned}
& \text { - dans } \mathbb{C}^{3}, \mathbb{O}_{\mathbb{C}^{3}, 0}=\mathbb{C}\{x, y, z\} \quad I^{1}=(x, y), I^{2}=(y, z), I^{3}=(x, z) \\
& \left(C^{1} ; c^{2} \cup c^{3}\right)=1 \text { tandis que }\left(c^{1} ; c^{2}\right)=\left(C^{1} ; c^{3}\right)=1 \\
& \text { - dans } c^{4}, C_{\mathbb{C}^{4}, 0}=\mathbb{C}\{x, y, z, w\} \quad I^{1}=(x-z, y-w, f) \\
& I^{2}=(x, y, g), I^{3}=(z, w, h) \text { où } f, g, h \text { sont des séries d'ordres supérieurs à } \\
& \text { deux, } \\
& \left(C^{1} ; C^{2} \cup c^{3}\right)=3 \text { tandis que }\left(C^{1} ; c^{2}\right)=\left(C^{1} ; C^{3}\right)=1 . \\
& \text { PROPOSITION II.7. }
\end{aligned}
$$

Soit $p: X \rightarrow U \subset \mathbb{C}$ une déformation plate centrée d'une courbe réduite $X_{0}$. on suppose que les $r$ composantes irréductibles $x^{1}, \ldots, x^{r}$ de $x$ ont pour fibres en 0 les $r$ composantes irréductibles de $X_{0}$. Alors les conditions suivantes sont équivalentes :

(i) $\mu\left(X_{u}\right)$ est constant

(ii) pour $j=1, \ldots, r \mu\left(x_{u}^{j}\right)$ et $\left(x_{u}^{j} ; x_{u}^{j+1} u \ldots u x_{u}^{r}\right)$ sont constants.

\section{PREUVE :}

(ii) $\Rightarrow$ (i) grâce au lemme I.2 et à la proposition II.5. (i) $\Rightarrow$ (ii) grâce à la proposition II.5, la semi-continuité de $\mu\left(X_{u}^{j}\right)$ conséquence du théorème II.4 et la semi-continuité de la colongueur, $\left(X_{u}^{j} ; x_{u}^{j+1} u \ldots u X_{u}^{r}\right)$.

\section{REMARQUE II.8. :}

La condition de la proposition II.7 n'est pas toujours réalisée dans une déformation à $\mu$ constant, voir l 'exemple V.1.

Une déformation plate centrée à $\mu$ constant $n$ 'est pas en générale équimultiple. L'exemple le plus simple est donné par la paramètrisation en famille $\left(x=t^{3}+u t^{2}, y=t^{4}, z=t^{5}\right)$; voir aussi l 'exemple V.1 du à J.P. Henry où $X_{0}$ est une intersection complète réductible et l'exemple V.2 où $X_{0}$ est de Gorenstein et irréductible. 


\section{CHAP, III : LES CONDITIONS DE WHITNEY}

Dans ce chapitre, nous allons étudier le critère d'équisingularité donné par les conditions de Whitney entre la partie lisse de $X$ et son lieu singulier Sing $(X)$ : Rappelons tout d'abord la définition de ces conditions dans la situation qui nous intéresse :

DEFINITION III.1. :

Soit $p: X \rightarrow U$ une déformation plate centrée d'un germe de courbe réduite $X_{0}$ de $\mathbb{a}^{N}$; cette déformation vérifie les conditions de Whitney au point 0 si, pour toute suite $M_{l}=\left(m_{\ell}, u_{\ell}\right) \in X-U \subset C^{N} \times \mathbb{C}$ tendant vers 0 , telle que 1a limite du plan tangent à $X$ en $M_{\ell}$ existe, soit $P$, et telle que la limite du vecteur unité $m_{\ell} /\left\|m_{\ell}\right\|$ existe, soit $\omega$, on a les propriétés :
a) $U \subset P$
b') $\omega \subset P$

DEEFINITION III.2. :

On dit que la déformation $p: X \rightarrow U$ vérifie les conditions de Whitney le long de $U$ au voisinage de 0 - ou les conditions de Whitney strictes en 0 - si les conditions de Whitney sont satisfaites dans un voisinage de 0 de $U$. Nous renvoyons le lecteur à ( $[W] \S 19,\left[\mathrm{H}_{1}\right] \S 3,\left[\mathrm{Ma}_{2}\right] \S 1$ ) pour les définitions générales des conditions de Whitney (et le fait que ces définitions sont indépendantes du plongement) et à ( $[S p]$ p. 225) pour l'analyticité des conditions de Whitney strictes. 


\section{DÍFORMATIONS ÍQUUISINGULIÈRES}

Nous allons montrer maintenant l'analogue, pour les familles de courbes, du théorème " $\mu^{*}$ constant $\Leftrightarrow$ conditions de Whitney" démontré dans le cas des familles d'hypersurfaces ([B-Sp] th. II.2.6) et $\left(\left[\mathrm{T}_{4}\right]\right)$.

THÉORËME III.3. :

Soit $p: X \rightarrow U$ une déformation plate centrée d'un germe de courbe réduite $x_{0}$ de $\mathbb{C}^{N}$; les deux assertions suivantes sont équivalentes :

(i) la déformation vérifie les conditions de Whitney le long de $U$

(ii) la multiplicité $m\left(X_{u}\right)$ et le nombre de Milnor $\mu\left(X_{u}\right)$ sont constants dans $U$.

\section{PREUVE :}

$(i) \Rightarrow(i i)$ : la constance de $m\left(X_{u}\right)$ résulte d'un théorème de H. Hironaka

( $\left[\mathrm{H}_{2}\right]$ cor. 6.2). D'autre part le théorème de Thom-Mather ([ $\left.\mathrm{Ma}_{2}\right]$ Prop. 11.1)

montre que, puisque la stratification $\left(U_{\varepsilon} B_{\varepsilon}-X, X-U, U\right)$ vérifie les conditions de Whitney, la paire $\left(U_{E} B_{\varepsilon}, X\right)$ est topologiquement triviale au dessus de $U: i l$ en résulte, d'après le théorème II.4 que $\mu\left(X_{u}\right)$ est constant.

(ii) $\Rightarrow(i)$ : en utilisant le théorème II.4, nous savons que $\delta\left(X_{u}\right)$ et $r=r\left(X_{u}\right)$ sont constants. Or $\delta\left(X_{u}\right)$ constant implique (théorème II.3) l'existence d'une paramètrisation en famille : X est l'image directe du morphisme $n: U \times \frac{\mathbb{H}^{\mathrm{H}}}{j=1}\left(\mathbb{C}_{j}, 0\right) \rightarrow U \times\left(\mathbb{C}^{N}, 0\right)$. Notons, pour $j=1 \ldots r, x^{j}$ 1a composante irréductible de $x$ image de $n_{j}: U \times\left(\mathbb{C}_{j}, 0\right) \rightarrow U \times\left(\mathbb{C}^{N}, 0\right)$. Comme la multiplicité de $X_{u}$ est constante, somme des multiplicités de chaque branche, et la multiplicité étant semi-continue dans une famille paramétrée, on sait que la multiplicité $m_{j}$ de $x_{u}^{j}$ est constante; alors, quitte à faire un changement de coordonnées, on peut choisir 1 'axe des $x_{1}$ tangent à $X_{0}^{j}$ pour $u$ appartenant à un voisinage de 0 et écrire $n_{j}$ sous la forme :

$$
\left\{\begin{array}{l}
x_{1}\left(u, t_{j}\right)=t_{j}^{m_{j}}\left(1+u h_{1}\left(u, t_{j}\right)\right) \\
x_{j}\left(u, t_{j}\right)=t_{j}^{l_{j}} \varphi_{j}\left(t_{j}\right)+u t_{j}^{m_{j}} h_{j}\left(u, t_{j}\right)
\end{array}\right.
$$


pour $i=2, \ldots, N, \ell_{i}>m_{j}, \varphi_{i} \in \mathbb{C}\left\{t_{j}\right\}, h_{i} \in \mathbb{C}\left\{u, t_{j}\right\}$

Prenons un point $M$ de $x^{j}-u$, de coordonnées $\left(u, x_{1}\left(u, t_{j}\right), \ldots, x_{N}\left(u, t_{j}\right)\right)$; quand $M$ tend vers 0, la limite du plan tangent à $M$ contient la limite des vecteurs

$\frac{1}{m_{j} t_{j}-1} \frac{\partial M}{\partial t_{j}}$ et $\frac{\partial M}{\partial u}$ (1orsque $t_{j}$ et $u$ tendent vers 0 ), c'est à dire les vecteurs $(0,1,0, \ldots, 0)$ et $(1,0, \ldots, 0,0)$.

La condition a) de Whitney en 0 est donc vérifiẹe ; pour la condition b'), remarquons que $\frac{1}{t_{j}}\left(x_{1}\left(u, t_{j}\right), \ldots, x_{N}\left(u, t_{j}\right)\right)$ tend vers $(1,0, \ldots, 0)$.

Enfin, nous aurions démontré de la même manière les conditions de Whitney en tout point de $U:$ (i) est donc démontré.

Nous allons maintenant rappeler le résultat correspondant de $H$. Stutz ( $[\mathrm{St}]$ prop. 1.13); pour cela donnons une définition : $C_{4}(x, 0)$ désigne le cône formé par les limites en 0 des vecteurs tangents à la partie lisse de $X$.

- THÉORËME III.4. (J. Stutz) :

Une déformation plate centrée $p: X \rightarrow U$ d'un germe de courbe réduite $x_{0}$ vérifie les conditions de Whitney strictes en 0 si et seulement $\operatorname{sid} \operatorname{dim}\left(C_{4}(X, 0)\right)=2$.

Le résultat de J. Stutz est plus génēral ; ici il s'applique directement puisque nous avons démontré dans la proposition I. 6 que toute composante irréductible de $X$ est essentielle.

Donnons à présent un "critère discriminant" pour les conditions de Whitney strictes, en nous limitant au cas des intersections complètes :

\section{THEOREMME III.5}

Soit $\mathrm{p}: \mathrm{X} \rightarrow U$ une déformation plate centrée d'un germe de courbe réduite $\mathrm{X}_{0}$, intersection complète dans $\mathbb{a}^{N}$. Les conditions suivantes sont équivalentes: 


\section{DÉFORMATIONS ÉQUISINGULIÈRES}

(i) la déformation $p: X \rightarrow U$ vérifie les conditions de Whitney strictes en 0 .

(ii) pour tout paramètre transverse $x$ de $x_{0}$, le lieu discriminant réduit de la projection de $x$ sur le plan $(u, x)$ est $l$ 'axe des $u$ au voisinage de 0 .

PREUVE : $(i) \Rightarrow$ (ii).

Quitte à faire un changement de coordonnées dans $c^{N}$ supposons que $x_{1}=x$.

D'après le théorème III.3, $\mu\left(X_{u}\right)$ et $m\left(X_{u}\right)$ sont constants dans $U$ et d'après la démonstration du théorème III.3 $((i i) \Rightarrow(i))$, il existe une paramétrisation en famille à multiplicité constante de chaque composante $x^{j}$ de $X$ :

$$
\left\{\begin{array}{l}
x_{1}\left(u, t_{j}\right)=t_{j}^{m_{j}}\left(1+u h_{1}\left(u, t_{j}\right)\right) \\
x_{i}\left(u, t_{j}\right)=t_{j}^{\ell_{j}} \varphi_{i}\left(t_{j}\right)+u t_{j}^{m_{j}} h_{i}\left(u, t_{j}\right)
\end{array}\right.
$$

pour $i=2, \ldots, N, l_{i} \geqslant m_{j}$ et $\left(u, t_{j}\right)$ dans un voisinage assez petit de $(0,0)$.

Le plan tangent à $X$ au point $M \in X^{j}-U$ de coordonnées $\left(u, x_{1}\left(u, t_{j}\right), \ldots x_{N}\left(u, t_{j}\right)\right)$

$t_{j} \neq 0$ est engendré par les vecteurs $\left(0, \partial x_{1} / \partial t_{j}, \ldots, \partial x_{N} / t_{j}\right)$ et

$\left(1, \frac{\partial x_{1}}{\partial u}, \ldots, \frac{\partial x_{N}}{\partial u}\right)$. Le point $M$ appartient au lieu critique Crsi et seulement si $\partial x_{1} / \partial t_{j}=m_{j} t_{j}^{m_{j}-1}\left(1+u h_{1}+t_{j} \frac{u}{m_{j}} \partial h_{1} / \partial t_{j}\right)=0$ ce qui entrainerait $t_{j}=0$ et $x_{1}=x=0$. Ainsi $x^{j}-U$ ne contient aucun point critique et le discriminant réduit de la projection de $X$ sur le plan des $(u, x)$ est égal à l'axe des $u$.

(ii) $\Rightarrow(i)$ : Notons $F_{1}, \ldots, F_{N-1}$ les fonctions analytiques au voisinage de 0 définissant $X$ et $\bar{\omega}$ la restriction à $X$ de la projection $\bar{\omega}\left(u, x_{1}, \ldots, x_{N}\right)=\left(u, x_{1}\right)$

de $\mathbb{C} \times \mathbb{C}^{N}$ sur $\mathbb{C}^{2}$.

Le lieu critique $C$ est défini par l'idéal

$J=\left(F_{1}, \ldots, F_{N-1}, \frac{\partial\left(F_{1}, \ldots, F_{N-1}\right)}{\partial\left(x_{2}, \ldots, x_{N}\right)}\right)$ où $\frac{\partial\left(F_{1}, \ldots, F_{N-1}\right)}{\partial\left(x_{2}, \ldots, x_{N}\right)}$ est le 
déterminant des dérivées partielles de $\mathrm{F}_{1}, \ldots, \mathrm{F}_{\mathrm{N}-1}$, par rapport aux coordonnées $x_{2}, \ldots, x_{N}$. La projection đ étant ffnie on peut définir le lieu discriminant par le $0^{i \text { ème }}$ idéal de Fitting du $\mathbf{C}\left\{u, x_{1}\right\}$-module de type fini $\bar{\omega}_{*}\left(\mathbf{C}\left\{u, x_{1}, \ldots, x_{N}\right\} / J\right)$. Puisque $C$ est une intersection complète donc de Cohen Macaulay, et de dimension 1, le $0^{i e ̀ m e}$ idéal de Fitting est principal $\left(\left[T_{1}\right]\right.$ § 3,5) engendré par $\Delta\left(u_{2} x_{1}\right)$. L'hypothèse $(i i)$ entraine alors que $\Delta\left(u, x_{1}\right)$ est égal à $x_{1}{ }^{{ }^{0}}$ à une unité près. Les idéaux de Fitting sont stables par changement de base donc pour $u=\underline{u}$ fixé assez petit $\Delta\left(\underline{u}, x_{1}\right)$ engendre le $0^{i e ̀ m e}$ idéal de Fitting du $\mathbb{C}\left\{x_{1}\right\}$-module de type fini $\mathbb{a}\left\{x_{1}, \ldots, x_{N}\right\} / J_{\underline{u}}$, au voisinage de 0 , oũ $\underline{J}_{\underline{u}}=n_{\underline{u}}^{*}(J)$ est 1 'image réciproque de J par l'injection $\eta_{\underline{u}}: x \rightarrow(\underline{u}, x)$ de $\mathbf{C}^{N}$ dans $\mathbf{C} \times \mathbf{C}^{N}$. La multiplicité de $\Delta_{1}\left(\underline{u}, x_{1}\right)$, dans l'anneau principal $\mathbf{C}\left\{x_{1}\right\}$ est égal à la colongueur :

$\operatorname{col}\left(\mathcal{J}_{\underline{u}}\right)=\operatorname{dim}_{\mathbb{C}}\left(\mathbb{C}\left\{x_{1}, \ldots, x_{n}\right\} / J_{\underline{u}}\right)\left(=\Delta_{0}\right.$ d'après $\left.(i i)\right)$.

D'autre part la formule de Lê Dũng Tráng et G.M. Grevel pour les intersection complètes ( $\left[\hat{e}_{1}\right]$, cor. 3.7.2) donne dans notre cas pour u=u fixé :

$\begin{aligned} \mu\left(\mathrm{X}_{\underline{u}}\right)+\mu\left(\mathrm{F}_{1}, \ldots, \mathrm{F}_{\mathrm{N}-1}, \mathrm{x}_{1}\right) & =\operatorname{col}\left(\mathrm{F}_{1}, \ldots, \mathrm{F}_{N-1}, \frac{\partial\left(\mathrm{F}_{1}, \ldots, \mathrm{F}_{N-1}, \mathrm{x}_{1}\right)}{\partial\left(\mathrm{x}_{1}, \ldots, \mathrm{x}_{N}\right)}\right) \\ & =\operatorname{col}\left(\mathrm{F}_{1}, \ldots, \mathrm{F}_{N-1}, \frac{\partial\left(\mathrm{F}_{1}, \ldots, \mathrm{F}_{N-1}\right)}{\partial\left(\mathrm{x}_{2}, \ldots, \mathrm{x}_{N}\right)}\right)=\Delta_{0}\end{aligned}$

En raison de la semi-continuité de $\mu$ on en déduit que $\mu\left(X_{u}\right)=\mu\left(X_{0}\right)$ et que $\mu\left(F_{1}, \ldots, F_{N-1}, x_{1}\right)$ qui est la multiplicité d'intersection en 0 de $x_{u}$ et de 1 'hyperplan $x_{1}=0$ est constant et égal à $\left(x_{0} ; x_{1}=0\right)=m\left(x_{0}\right)-1 \quad\left(x_{1}\right.$ paramètre transverse à $x_{0}$ ). Si on choisit le paramètre $x_{1}=x$ de telle sorte que l'hyperplan $x_{1}=0$ ne contienne aucune limite de tangentes aux points singuliers de $x_{\underline{u}}$ lorsque $\underline{u} \rightarrow 0$, 1 'hyperplan $x_{1}=0$ est transverse ã $x_{\underline{u}}$ pour tout $\underline{u}$ et $\left(x_{\underline{u}} ; x_{1}=0\right)=m\left(x_{\underline{u}}\right)-1$ est donc constant. 


\section{DÉFORMATIONS ÉQUISINGULIÈRES}

REMARQUE III.6.

L'implication $(i) \Rightarrow(i i)$ du théorème précédent est vraie sans l'hypothèse restrictive que $x_{0}$ soit une intersection complète. La démonstration que nous donnons de l'implication (ii) $\Rightarrow$ (i) ne se généralise pas car elle utilise le fait que pour une courbe $x_{0}$ intersection complète ( $i i$ ) implique que le discriminant de la projection générique est équimultiple ce qui est faux dans le cas général comme le montre l'exemple V.5.

Revenons au cas général et comparons l'équisingularité par les conditions de Whitney avec la résolution simultanée forte définie par $B$. Teissier $\left(\left[\mathrm{T}_{2}\right]\right.$, définition 3.1.5) :

DÉFINITION III.7.

$p: X \rightarrow U$ admet une résolution simultanée forte si $p$ admet une résolution simultanée faible $n: \bar{X} \rightarrow X$ (cf. II.2) et si de plus le morphisme induit par restriction pon : $n^{-1}(U) \rightarrow U$ est simple.

THÉORÈME III.8. : (B. Teissier).

Soit $p: X \rightarrow U$ une déformation plate centrée d'un germe de courbe réduite $x_{0}$ de $a^{N}$; les conditions suivantes sont équivalentes :

(i) $p: X \rightarrow U$ vérifie les conditions de Whitney le long de $U$.

(ii) $p: X \rightarrow U$ admet une résolution simultanée forte.

PREUVE :

(i) $\Rightarrow$ (ii) :

Par le théorème III.3 nous savons que $\mu\left(X_{u}\right)$ et $m\left(X_{u}\right)$ sont constants, et par le théorème II.3 qu'il existe unerésolution simultanée faible ; nous pouvons écrire au voisinage de 0 :

$$
n: U \times \underset{j=1}{\stackrel{r}{H}}\left(\mathbb{C}_{j}, 0\right) \rightarrow U \times\left(\mathbb{C}^{N}, 0\right)
$$


$\operatorname{avec} n_{j}\left(u, t_{j}\right)=\left(u, x_{1}\left(u, t_{j}\right), \ldots, x_{N}\left(u, t_{j}\right)\right)$

et l'image réciproque de $U$ par $n_{j}$ est définie, au voisinage de 0 par l'idēal

de $a\left\{u, t_{j}\right\}$ engendré par $x_{1}\left(u, t_{j}\right), \ldots, x_{N}\left(u, t_{j}\right)$.

Mais comme la multiplicité est constante, égale à $m_{j}$, cet idéal n'est autre que $\left(t_{j}^{m_{j}}\right)$ et la restriction de $p \circ n_{a} n^{-1}(U)$ est donc simple

(ii) $\Rightarrow$ (i) :

est démontré de manière générale par $B$. Teissier $\left(\left[_{2}\right]\right.$ proposition 4$)$. 


\section{a) Définition de la projection plane générique :}

On considère un germe de courbe réduite $x_{0}$ dans $\left(\mathbb{C}^{N}, 0\right)$; nous allons commencer par préciser le cône $C_{5}\left(X_{0}, 0\right)$ ensemble des limites des vecteurs sécants à $X_{0}$ : un vecteur $\vec{\omega}$ appartient à $C_{5}\left(X_{0}, 0\right) s^{\prime} i l$ existe deux suites de points $P_{n}$ et $Q_{n}$ de $x_{0}$ tendant vers 0 et une suite $\lambda_{n}$ de nombre complexes telles que

$$
\vec{w}=\lim _{n \rightarrow \infty}\left(\lambda_{n} \vec{P}_{n} Q_{n}\right) \text {. }
$$

\section{PROPOSITION IV.1. :}

Si $x_{0}$ est singulière, $c_{5}\left(x_{0}, 0\right)$ est la réunion d'un nombre fini de 2-plans, chacun d'eux passant par une tangente à $X_{0}$.

\section{PREUVE :}

Il suffit d'étudier les limites dans $\mathbb{P}^{1}\left(\mathbb{C}^{\mathrm{N}}\right)$ des directions de droites $\left(M_{k_{1}}(t(s)), M_{k_{2}}\left(t^{\prime}(s)\right)\right)$ lorsque $s$ tend vers zéro, pour tous les germes de fonctions analytiques $t(s)$ et $t^{\prime}(s)$ de $(\boldsymbol{C}, 0)$ vers $(\boldsymbol{C}, 0)$ tels que $M_{k_{1}}(t(s)) \neq M_{k_{2}}\left(t^{\prime}(s)\right)$, $M_{k}:(\mathfrak{C}, 0) \rightarrow x_{0}^{k}$ étant une paramètrisation de la k-ième branche de $x_{0}$. Si $x_{0}^{k_{1}}$ et $x_{0}^{k_{2}}$ ont des tangentes distinctes on obtient ainsi toutes les directions du 2-plan défini par ces tangentes; nous supposons donc que $x_{0}^{k_{1}}$ et $x_{0}^{k_{2}}$ ont pour tangente commune l'axe des $x_{1}$. Notons $n$ le ppcm des multiplicités des branches $x_{0}^{k_{1}}$ et $x_{0}^{k_{2}}$ qui admettent des paramétrisations de la forme :

$$
\left\{\begin{array} { l } 
{ x _ { 1 } ( t ) = t ^ { n } } \\
{ x _ { j } ( t ) = \sum _ { i > n } a _ { j , i } t ^ { i } }
\end{array} \quad \left\{\begin{array}{l}
x_{1}^{\prime}\left(t^{\prime}\right)=t^{\prime n} \\
x_{j}^{\prime}\left(t^{\prime}\right)=\sum_{i>n} b_{j, i} t^{\prime i}
\end{array}\right.\right.
$$

pour $j=2, \ldots, N$. 
Supposons $v\left(t^{\prime}\right) \leq v(t), v$ désignant la valuation naturelle de $\mathbb{C}\{s\}$.

Notons $: \alpha(s)=(t(s))^{n}-\left(t^{\prime}(s)\right)^{n}, \gamma_{j}(s)=x_{j}(t(s))-x_{j}^{\prime}\left(t^{\prime}(s)\right)$

pour $j=2, \ldots, N$, et posons $\varepsilon=\lim \frac{t(s)}{t^{\prime}(s)}, v_{0}=v(t(s)), v_{1}=v\left(t(s)-\varepsilon t^{\prime}(s)\right)$.

La 1 imite de la direction sécante $\left(\alpha(s), \gamma_{2}(s) \ldots, \gamma_{N}(s)\right)$ est l'axe des $x_{1}$ dans les deux cas suivants, où

$v_{1}+(n-1) v_{0}=v(\alpha)<v\left(\gamma_{j}\right)$ pour $j=2, \ldots, N$ :

1) Torsque $\varepsilon^{n} \neq 1$

2) lorsque $\varepsilon^{n}=1$ et, pour tout $j=2, \ldots, N$ et $i>n$

$$
\varepsilon^{i} a_{j, i}-b_{j, i}=0 \text {. }
$$

Il reste à étudier le cas où $\varepsilon^{n}=1$ et où

$k=\inf \left\{i / \exists j b_{j, i} \neq \varepsilon^{i} a_{j, i}\right\}$ est un entier fini. Remarquons alors que

$v\left(a_{j, i} t^{i}-b_{j, i} t^{\prime i}\right)>v(\alpha)$ lorsque $i<k, v\left(a_{j, i} t^{i}-b_{j, i} t^{\prime i}\right) \geqslant i v_{0}>k v_{0}$

lorsque $i>k$ et enfin que $v\left(a_{j, k} t^{k}-b_{j, k} t^{\prime k}\right)=k v_{0}$ lorsque

$a_{j, k} \varepsilon^{k} \neq b_{j, k}$.

Notons $\vec{v}=\left(0, \varepsilon^{k} a_{2, k}-b_{2, k}, \ldots, \varepsilon^{k} a_{N, k}-b_{N, k}\right)$ et $\vec{e}_{1}=(1,0, \ldots, 0)$;

$i 1$ résulte du calcul précédent que la direction $\left(\alpha(s), \gamma_{2}(s), \ldots, \gamma_{N}(s)\right)$ admet pour limite la direction suivante :

- l'axe des $x_{1}$ si $v(\alpha)<k v_{0}$

- la direction de $\vec{v}$ si $v(\alpha)>k v_{0}$

- la direction du vecteur $\vec{v}+\lambda \vec{e}_{1}$ si $v(\alpha)=k v_{0}$ et si

$$
\lim \frac{\alpha(s)}{t^{\prime}(s)^{k}}=\lambda \text {. }
$$

Quel que soit $\lambda \in \mathbb{C}-\{0\}$, on peut trouver $t(s)$ et $t^{\prime}(s)$ tels que 


\section{DÉFORMATIONS ÉQUISINGULIÈRES}

$v_{1}=v\left(t-\varepsilon t^{\prime}\right)=(k-(n-1)) v_{0}$ et $\alpha(s) \sim \lambda t^{\prime}(s)^{k}$.

L'ensemble des limites de sécantes obtenues pour $\varepsilon$ fixé (tel que $\varepsilon^{n}=1$ ) est donc l'ensemble des directions de droites du plan engendré par l'axe des $x_{1}$ et le vecteur $\vec{v}$.

Soit $\mathcal{G}$ la grassmannienne des $(N-2)$-plans de $\mathbb{C}^{N}$; pour $H \in \mathscr{G}$ notons $\mathbb{I}_{H}$ la projection linéaire parallèlement à $H$ sur un 2-plan transverse ; lorsque $0 \in C^{N}$ est isolé dans $H \cap X_{0}$, la restriction de $\pi_{H}$ à $X_{0}$ est finie et $\pi_{H}\left(X_{0}\right)$ est un germe de courbe plane.

\section{-PROPOSITION IV.2. :}

Soit $\Omega$ l'ouvert dense de Zariski de $\mathscr{G}$ ensemble des (N-2)-plans de $\mathbb{C}^{N}$ ne contenant aucune limite de sécantes à $X_{0}$ ( $c^{\prime}$ est à dire transverses à $C_{5}\left(X_{0}, 0\right)$ );

a) Pour $H \in \Omega, O$ est isolé dans $H \cap X_{0}, \Pi_{H}\left(X_{0}\right)$ est réduite et à type topologique constant (on note $\mu_{0}^{\prime}$ le nombre de Milnor constant)

b) Pour $H \notin \Omega$, l'une des assertions suivantes est vérifiée :

- O n'est pas isolé dans $H$ H $_{0}$

- 0 est isolé dans $H \cap X_{0}$ et $\pi_{H}\left(X_{0}\right)$ n'est pas réduite.

- 0 est isolé dans $H \cap X_{0}, \pi_{H}\left(X_{0}\right)$ est réduite et son nombre de Milnor est strictement plus grand que $\mu_{0}^{\prime}$.

\section{PREUVE :}

Soit $W$ l'ouvert de $\mathscr{G}$ formé par les $(\mathrm{N}-2)$-plans de $\mathbb{C}^{N}, H$, tels que $0 \in \mathbb{C}^{N}$ soit isolé dans $H \cap X_{0}$; si les formes linéaires $x_{=} a_{1} x_{1}+\ldots a_{N} x_{N}$ et $y=b_{1} x_{1}+\ldots+b_{N} x_{N}$ sont indépendantes, on note $H_{d}$ le $(N-2)-p l a n$ d'équations $x=y=0$ pour $d=(a, b) \in \mathbb{C}^{2 N}$; et on note $W^{\prime} 1$ 'ouvert de $\mathbb{C}^{2 N}$ défini par la condition : $d \in W^{\prime}$ si $H_{d} \in W$. Soit $\pi_{d}: \mathbb{C}^{N} \rightarrow \mathbb{C}^{2}$ la projection linéaire $\pi_{d}\left(x_{1}, \ldots, x_{N}\right)=(x, y)$ parallèlement à $H_{d}$; si $d \in W^{\prime}$, la restriction de $\pi_{d}$ à $X_{0}$ est finie, et on note 
$\Pi_{d}\left(x_{0}\right)$ son image, définie par le $0^{i e ̀ m e}$ idéal de Fitting $d u \mathbb{C}\{x, y\}$-module $\pi_{d_{*}}\left(\mathcal{O}_{x_{0}}\right)$. Cet idéal est principal $\left(\left[T_{1}\right] \S 3.5\right)$ et définit ensemblistement 1 'image de $X_{0} \cap V_{d}$ par $\pi_{d}, V_{d}$ étant un voisinage assez petit de 0 dans $\mathbb{C}^{N}$. Considérons le morphisme $\pi: \mathbb{C}^{N} \times \mathbb{C}^{2 N} \rightarrow \mathbb{C}^{2} \times \mathbb{C}^{2 N}$ dont les fibres sont les projections $\pi_{d}$ :

$$
\pi\left(x_{1}, \ldots, x_{N} ; d\right)=\left(\pi_{d}\left(x_{1}, \ldots, x_{N}\right) ; d\right)
$$

La restriction de $\pi$ à $X_{0} \times W^{\prime}$ est finie en $(0, \underline{d})$ dès que $\underline{d} \in W^{\prime} ; l^{\prime}$ 'anneau local $\mathcal{O}=\mathcal{O}_{X_{0} \times W^{\prime},(0, \underline{d})}$ étant de Cohen Macaulay de dimension $2 N+1$, le $0^{i e ̀ m e}$ idéal de Fitting de $\pi_{*}(\mathcal{O})$ qui définit $l^{\prime}$ image $\pi\left(X_{0} \times W^{\prime},(0, \underline{d})\right)$ de ce germe est principal. Le morphisme $G: \Pi\left(X_{0} \times W^{\prime},(0, \underline{d})\right) \rightarrow\left(W^{\prime}, \underline{d}\right)$ est donc une déformation plate de sa fibre au dessus de $\underline{d}, G^{-1}(\underline{d}) \approx \Pi_{\underline{d}}\left(X_{0}\right)$. Soit $M_{k}:(\mathbb{C}, 0) \rightarrow x_{0}^{k}$ la normalisation de la $k^{i e ̀ m e}$ branche de $x_{0}^{k}$, $M_{k}\left(t_{k}\right)=\left(x_{1}\left(t_{k}\right), \ldots, x_{N}\left(t_{k}\right)\right) ; 1$ a déformation plate $G$ admet une paramètrisation en famille $x\left(t_{k}\right)=a_{1} x_{1}\left(t_{k}\right)+\ldots a_{N} x_{N}\left(t_{k}\right), y\left(t_{k}\right)=b_{1} x_{1}\left(t_{k}\right)+\ldots+b_{N} x_{N}\left(t_{k}\right)$.

PREUVE de a): lorsque $H_{d}$ ne contient aucune limite de sécantes à $X_{0}$, pour tout $d$ voisin de $\underline{d}, \Pi_{d}: X_{0}-\{0\} \rightarrow G^{-1}(d)-\{0\}$ est un isomorphisme, et donc $G$ est une déformation plate centrée à $\delta\left(G^{-1}(d)\right)$-constant et $r\left(G^{-1}(d)\right)=r\left(X_{0}\right)$-constant ; donc à $\mu\left(G^{-1}(d)\right.$ ) constant (théorème II.4).

PREUVE de b) : nous supposons que $H_{d}$ contient une limite de sécantes à $X_{0}$, que 0 est isolé dans $H_{d} \cap X_{0}$ et que $\pi_{\underline{d}}\left(X_{0}\right)$ est réduite; $G$ est une déformation plate de la courbe plane $G^{-1}(d)$, et pour une suite $d_{n}$ de points tendant vers $d$, il existe une sécante $P_{n} Q_{n}$ à $X_{0}$;

a) si $\pi_{d_{n}}\left(P_{n}\right)=\pi_{d_{n}}\left(Q_{n}\right)$ est distinct de $0, G^{-1}\left(d_{n}\right)$ a en ce point une singularité et par conséquent $\mu\left(G^{-1}\left(d_{n}\right), 0\right)<\mu\left(G^{-1}(d), 0\right)$; 
B) si $\pi_{d_{n}}\left(P_{n}\right)=\pi_{d_{n}}\left(Q_{n}\right)=0$, le nombre de branches de $G^{-1}\left(d_{n}\right)$ en 0 est strictement supérieur à $r\left(G^{-1}(\underline{d})\right)=r\left(X_{0}\right)$ et on arrive à la même conclusion qu'en $\alpha$ ) pour les nombres de Milnor.

Dans les deux cas, $\mu\left(G^{-1}(\underline{d}), 0\right)>\mu\left(G^{-1}\left(d_{n}\right), 0\right) \geq \mu_{0}^{\prime}$, 1a dernière inégalité provenant de la semi-continuité du nombre de Milnor.

DÉFINITION IV.3. :

On appelle projection plane générique de $x_{0}$ le germe de courbe plane $X_{0}^{\prime}=\pi_{H}\left(X_{0}\right), H \in \Omega$, défini à équivalence topologique près.

Nous montrons dans $T^{\prime}$ 'appendice VI que $\tilde{\mathcal{O}}_{X_{0}} \approx \tilde{\mathcal{O}}_{X_{0}^{\prime}}$, où $\hat{\mathcal{X}}_{Y}$ désigne le saturé de 1 'anneau du germe de courbe $Y$; et que, si $Y_{1}$ et $Y_{2}$ sont des courbes planes, $\partial_{Y_{1}} \approx \mathscr{\gamma}_{Y_{2}}$ si et seulement si $Y_{1}$ et $Y_{2}$ sont topologiquement équivalentes. Il en résulte que la projection plane générique de $X_{0}$ est un invariant analytique de $X_{0}$. b) Définition et semi continuité de l'invariant $\ell\left(X_{0}\right)$ :

DEFINITION IV.4. :

Soient : $X_{0}$ un germe de courbe réduite dans $\left(\mathbb{C}^{N}, 0\right), X_{0}^{1}$ sa projection plane générique, $\delta_{0}=\delta\left(X_{0}\right), \mu_{0}=\mu\left(X_{0}\right), \delta_{0}^{\prime}=\delta\left(X_{0}^{\prime}\right)$ et $\delta_{0}^{\prime}=\delta\left(X_{0}^{\prime}\right)$ leurs invariants respectifs; on pose :

$$
\ell\left(X_{0}\right)=\delta_{0}^{\prime}-\delta_{0}=\frac{1}{2}\left(\mu_{0}^{\prime}-\mu_{0}\right)
$$

REMARQUE IV.5.

$\delta_{0}^{\prime}$ et $\delta_{0}$ sont des invariants analytiques de $X_{0}$ ( $d^{\prime}$ après les remarques précédentes en ce qui concerne $\left.\delta_{0}^{\prime}\right)$ donc également $\ell\left(X_{0}\right) \cdot \ell\left(X_{0}\right)$ possède également la propriété évidente suivante $: \ell\left(X_{0}\right)=0$ si et seulement si $x_{0}$ est isomorphe à une courbe plane $\left(\operatorname{car} \ell\left(X_{0}\right)=\operatorname{dim} 0_{X_{0}} 10_{X_{0}^{\prime}}\right)$.

Montrons maintenant que l'invariant $\ell$ est semi-continu supérieurement : 


\section{PROPOSITION IV.6. :}

Soit $p: X \rightarrow U$ une déformation plate d'un germe de courbe réduite $X_{0}$. On note $\ell\left(X_{u}\right)=\sum_{x \in \operatorname{Sing}\left(X_{u}\right)} \ell\left(X_{u}, x\right)$.

Alors $\ell\left(X_{u}\right) \leq \ell\left(X_{0}\right)$. Plus précisément, si $\operatorname{sing}\left(x_{u}^{\prime}\right)=\left\{y_{1}, \ldots, y_{k}\right\}$ et $n_{i}=\# \pi_{d}^{-1}\left(y_{j}\right)$ pour $i=1, \ldots, k$, où $\Pi_{d}: x_{0} \rightarrow x_{0}^{\prime}$ est une projection plane générique et $X_{u}^{\prime}=\pi_{d}\left(X_{u}\right)$, on $a:$

$$
\ell\left(x_{0}\right)-\ell\left(x_{u}\right) \geq \frac{1}{2} \quad \sum_{i=1}^{k} n_{i}\left(n_{i}-1\right) .
$$

\section{PREUVE :}

Soit $\pi_{d}: X_{0} \rightarrow X_{0}^{\prime}$ une projection générique; le $(N-2)-p l a n H_{d}$ de $\mathbb{C}^{N}$ est transverse à $x_{0}$ et donc $\pi=\pi_{d} \times$ Id $: X \rightarrow \mathbb{C} \times a^{2}$ est une projection finie dont $I^{\prime} i$ mage $\Pi(X)=X^{\prime}$ définie par le 0 -iàme idéal de Fitting de $\Pi_{*}\left(\mathcal{C}_{X}\right)$ est réduite, et le morphisme $p^{\prime}: X^{\prime} \rightarrow \mathbb{C}$ est une déformation plate de $X_{0}^{\prime}$.

Soit $n: \bar{X} \rightarrow X$ la normalisation de $X$. Le morphisme $\pi$ on $: \bar{X} \rightarrow X$ ' est aussi

la normalisation de $X^{\prime}$. Soit $(\bar{X})_{0}$ la fibre au dessus de 0 du morphisme $\bar{p}=p_{\circ}=p^{\prime} \circ \pi_{\circ} n: \bar{X} \rightarrow \mathbb{C}$; d'après le théorème de $B$. Teissier $\left(\left[\mathrm{T}_{1}\right]\right.$ p. 61), on $a:$

$$
\begin{aligned}
& \delta\left((\bar{x})_{0}\right)=\delta_{0}-\quad \sum_{x} \equiv \operatorname{sing}\left(x_{u}\right) \delta\left(x_{u}, x\right) \\
& =\delta_{0}^{\prime}-\quad \sum_{y \in \operatorname{Sing}\left(X_{u}^{\prime}\right)} \delta\left(X_{u}^{\prime}, y\right)
\end{aligned}
$$

En notant $\operatorname{sing}\left(x_{u}^{\prime}\right)=\left\{y_{1}, \ldots, y_{k}\right\}, \pi_{d}^{-1}\left(y_{i}\right)=\left\{x_{i, 1}, \ldots, x_{i, n}\right\}$ on a: $\left(x_{u}^{\prime}, y_{i}\right)=\underset{1 \leq j \leq n_{i}}{u} x_{u, i, j}^{\prime}$ où $x_{u, i, j}^{\prime}=\pi_{d}\left(x_{u}, x_{i, j}\right)$.

Donc si on remarque que $\operatorname{sing}\left(X_{u}^{1}\right) \supset \pi_{d}\left(\operatorname{sing} X_{u}\right)$ :

$$
\begin{aligned}
& \delta\left(x_{u}^{\prime}, y_{i}\right)=\sum_{j=1} \delta\left(X_{u, i, j}^{\prime}\right)+1 \leq j<j^{\prime} \leq n_{i}\left(x_{u, i, j}^{\prime} ; X_{u, i, j^{\prime}}^{\prime}\right) \\
& \delta\left(x_{u}^{\prime}, y_{j}\right) \geq \sum_{j=1}^{n_{j}} \delta\left(x_{u, i, j}^{\prime}\right)+\frac{1}{2} n_{j}\left(n_{j}-1\right)
\end{aligned}
$$


En reportant on obtient :

$\ell\left(X_{0}\right)=\delta_{0}^{\prime}-\delta_{0}=\sum_{y \in \operatorname{Sing}\left(X_{u}^{\prime}\right)} \delta\left(X_{u}^{\prime}, y\right)-\sum_{x \in \operatorname{Sing}\left(X_{u}\right)} \delta\left(X_{u}, x\right)$
$\ell\left(X_{0}\right) \geq \sum_{x \in \operatorname{Sing}\left(X_{u}\right)}\left[\delta\left(\pi_{d}\left(X_{u}, x\right)\right)-\delta\left(X_{u}, x\right)\right]+\frac{1}{2} \sum_{i=1}^{k} n_{i}\left(n_{i}-1\right)$

Et, puisque $\delta\left(\pi_{d}\left(X_{u}, x\right)\right)$ est au moins égal au $\delta$ de la projection générique du germe $\left(x_{u}, x\right):$

$\ell\left(x_{0}\right) \geq \ell\left(x_{u}\right)+\frac{1}{2} \sum_{i=1}^{k} n_{i}\left(n_{i}-1\right)$

COROLLAIRE IV.7. :

Soi $e^{r} p: x \rightarrow \mathbb{C}$ une déformation plate d'une courbe réduite $x_{0}$ et

$\Pi_{d}: X_{0} \rightarrow X_{0}^{\prime}$ une projection générique telles que pour $u \neq 0, X_{u}$ soit lisse,

et $X_{u}^{\prime} n^{\prime}$ ait que des points doubles ordinaires. Le nombre de ces points doubles ne dépend que de $x_{0}$ et est égal à $\ell\left(x_{0}\right)$.

PREUVE :

Il résulte de la démonstration de IV.6 que l'égalitẹ

$\ell\left(X_{0}\right)=\ell\left(X_{u}\right)+\sum_{i=1}^{k} \frac{1}{2} n_{i}\left(n_{i}-1\right)$ équivaut aux conditions suivantes:

1) $-\left(X_{u, i, j}^{\prime} ; X_{u, i, j^{\prime}}^{\prime}\right)=1$ pour $j \neq j^{\prime}$, ce qui entraine dans le cas où $n_{j}>1$

que $y_{i} \notin \pi_{d}\left(\operatorname{Sing}\left(x_{u}\right)\right)$ et que le germe $\left(x_{u}^{1}, y_{i}\right)$ est formé de $n_{j}$ branches lisses à tangentes distinctes.

2) - Si $x \in \operatorname{Sing}\left(x_{u}\right)$ et $y=\pi_{d}(x), \delta\left(\pi_{d}\left(x_{u}\right), y\right)=\delta^{\prime}\left(x_{u}, x\right)$ c'est à dire que la projection $\pi_{d}$ est générique pour le germe de $x_{u}$ en $x$.

3) - Si $x \notin \operatorname{Sing}\left(x_{u}\right)$ et $\pi_{d}(x)=y, \pi_{d}\left(x_{u}, x\right)$ est lisse en $y$.

Ces trois conditions sont satisfaites sous les conditions du corollaire avec

$n_{i}=2, \ell\left(x_{u}\right)=0$ donc :

$\ell\left(X_{0}\right)=\sum_{i=1}^{k} \frac{1}{2} n_{j}\left(n_{j}-1\right)=k$. 


\section{c) Équisaturation.}

\section{DEFINITION IV.8. :}

Une déformation plate centrée $p: X \rightarrow U$ d'un germe de courbe plane réduite $X_{0}$ est dite ésuisaturée en 0 si $\widetilde{O}_{x_{u}} \approx \widetilde{\mathscr{O}}_{x_{0}}$ au voisinage de 0 .

THEEOREME IV.8. :

Soit $p: X \rightarrow U$ une déformation plate centrée d'un germe de courbe plane $x_{0}$. Les conditions suivantes sont équitvalentes :

(i) La déformation plate $p$ est équisaturée en 0

(ii) Les entiers $\mu\left(X_{u}, 0\right)$ et $\ell\left(X_{u}, 0\right)$ sont constants dans un voisinage de $u=0$.

(iii) Il existe un ouvert dense $\Omega$ de $\mathscr{G}$ tel que si $H_{d} \in \Omega \quad p^{\prime}: \Pi_{d}(X) \rightarrow U$ est, au voisinage de $u=0$, une famille équisingulière de courbes planes.

PREUVE :

(i) $\Rightarrow$ (ii) Soit $X_{u}^{\prime}$ une projection générique de $\left(X_{u}, 0\right)$. Il résulte de 1 'appendice que $\widetilde{\mathcal{O}}_{X_{u}} \approx \widetilde{\mathscr{Q}}_{X_{u}^{\prime}}$ donc $\widetilde{\mathcal{O}}_{X_{u}^{\prime}} \approx \widetilde{\mathcal{O}}_{X_{0}^{\prime}}$, et que $X_{u}^{\prime}$ et $X_{0}^{\prime}$ sont topologiquement équivalentes.

Donc $\mu\left(X_{u}^{\prime}, 0\right)=\mu\left(X_{0}^{\prime}, 0\right)$ est constant. Comme $\mu\left(X_{u}^{\prime}, 0\right)=\mu\left(X_{u}, 0\right)+\ell\left(X_{u}, 0\right)$,

(ii) résulte alors de la semi continuité de $\mu\left(X_{u}\right)$ et de $\ell\left(X_{u}\right)$ (IV.6).

(ii) $\Rightarrow$ (iii) Soit $\Omega_{0} \subset \mathscr{G}$ l'ouvert des directions de projections płanes génériques de $X_{0}$. Si $H_{d} \in \Omega_{0}, \Pi_{d}(X)$ est une déformation plate de $X_{0}^{1}=\Pi_{d}\left(X_{0}\right)$ et on a :

$\mu_{0}^{\prime}=\mu\left(X_{0}^{\prime}\right) \geq \mu\left(\pi_{d}\left(X_{u}\right)\right) \geq \mu\left(X_{u}^{\prime}\right)=\mu_{0}^{\prime}$

Il en résulte que $\mu\left(\Pi_{d}\left(X_{u}\right)\right)=\mu_{0}^{\prime}$ est constant donc que $\pi_{d}(x)$ est une famille équisingulière de courbes planes.

(iii) $\Rightarrow$ (i) Soit $\Omega_{u}$ 1'ouvert des directions de projections génériques de $\left(X_{u}, 0\right)$ Si $H_{d} \in \Omega \cap \Omega_{0} \cap\left(\bigcap_{n=0}^{\infty} \Omega_{u_{n}}\right)$ où $u_{n}$ est une suite tendant vers zëro on a, pour $n$ assez grand. : 
$\mu\left(\Pi_{d}\left(X_{u_{n}}\right)\right)=\left(\mu\left(\pi_{d}\left(X_{0}\right)\right)=\mu_{0}^{\prime} d^{\prime}\right.$ 'après (iii).

D'autre part $\mu_{u}^{\prime}=\mu\left(X_{u}^{\prime}, 0\right)$ est semi continu supérieurement donc :

$\mu_{u_{n}}^{\prime} \leq \mu_{0}^{\prime}=\mu\left(\pi_{d}\left(x_{u_{n}}\right)\right) \leq \mu_{u_{n}}^{\prime}$ et $\mu_{u_{n}}^{\prime}=\mu_{0}^{\prime}$

Il en résulte que $\mu_{u}^{\prime}=\mu\left(\pi_{d}\left(x_{u}\right)\right)$ pour $u$ voisin de $u=0$, donc que $\pi_{d}\left(x_{u}\right)=x_{u}^{\prime}$ est une projection générique et $\pi_{d}(X)$ une famille équisinguliēre de courbes planes ; Ainsi :

$\widetilde{\mathscr{O}}_{\mathrm{X}_{u}} \approx \tilde{\mathfrak{O}}_{\pi_{d}}\left(\mathrm{x}_{\mathrm{u}}\right) \approx \widetilde{\partial}_{\mathrm{x}_{0}} \approx \widetilde{\partial}_{\mathrm{x}_{0}}$ et $\mathrm{p}$ est équisaturée en 0 .

- COROLLAIRE IV.9. :

Si $p: X \rightarrow U$ est une déformation plate centrée équisaturée en 0 , elle satisfait aux conditions de Whitney le long de $U$ au voisinage de 0 .

\section{PREUVE :}

D'après IV.8 si p est équisaturé $\mu\left(X_{u}\right)$ est constant dans $U$. D'autre part la multiplicité $m\left(X_{u}\right)$ de la courbe $X_{u}$ en 0 est égale à celle de l'anneau saturé $\mathcal{O}_{X_{u}}$ d'après l'appendice (th. VI.2.1) Rem. 2).

Donc $m\left(X_{u}\right)$ est constant dans $U$ et les conditions de Whitney résultent du théorème III.3.

Signalons encore le résultat suivant de J. Stutz ([St] proposition 3.14): THÉORËME IV.10 (J. Stutz) :

La déformation $p: X \rightarrow U$ est équisaturée en 0 si et seulement $\operatorname{si} \operatorname{dim}\left(C_{4}(X, 0)\right)=2$ et $\operatorname{dim}\left(C_{5}(x, 0)\right)=3$.

d) Cas des germes de courbes gauches intersections complètes dans $\mathbb{a}^{3}$.

Dans ce cas particulier, on peut préciser l'interprétation de l'invariant $\ell\left(X_{0}\right)$ comme nombre de points doubles de la projection générique d'une déformation lisse de $x_{0}$ et le calculer comme colongueur d'un idéal. 


\section{PROPOSITION IV.11 :}

Soient $x_{0}$ une courbe $d^{\prime}$ intersection complète de $c^{3}$, $d$ une direction de $c^{3}$ telle que la projection $\pi_{d}\left(X_{0}\right)$ de $X_{0}$ parallélement à $d$ sur un plan transverse soit à singularité isolée ;

a) il existe une déformation $p: X \rightarrow U$ de $x_{0}\left(X \subset U \times \mathbb{C}^{3}\right)$ telle que, pour $u$ non nul assez petit, la fibre générique $X_{u}$ soit lisse et $\pi_{d}\left(X_{u}\right)$ n'ait que des points doubles ordinaires.

b) le nombre $\ell_{d}$ de ces points doubles est $\delta\left(\pi_{d}\left(X_{0}\right)\right)-\delta\left(X_{0}\right)$.

\section{PREUVE :}

Dans le cas où $\pi_{d}$ est générique, on retrouve le résultat du corollaire IV.7. Dans le cas d'une direction de projection quelconque, l'assertion b) se démontre de la même façon.

Nous démontrons donc seulement la partie a).

On suppose que $(x, y, z)$ est un système linēaire de coordonnées tel que $d$ soit

la direction de l'axe de $z$ (et $\left.\Pi_{d}(x, y, z)=(x, y)\right)$.

Notons $f(x, y, z)=0$ et $g(x, y, z)=0$ des équations de $x_{0}$ et $\gamma=\inf \{v(f(0,0, z)) ; v(g(0,0, z))\}$ (v désignant la valuation naturelle de $\mathbb{C}\{z\}$ ) et définissons :

$$
\left\{\begin{array}{c}
F(x, y, z, a, b)=f(x, y, z)+\sum_{i=0}^{\gamma-1} a_{i} z^{i} \\
G(x, y, z, a, b)=g(x, y, z)+\sum_{i=0}^{\gamma-1} b_{i} z^{i}
\end{array}\right.
$$

$$
a=\left(a_{0}, a_{1}, \ldots, a_{\gamma-1}\right) \text { et } b=\left(b_{0}, b_{1}, \ldots, b_{\gamma-1}\right) \text {. }
$$

Le sous espace $x$ de $\mathbb{C}^{3} x \mathbb{a}^{2 \gamma}$ défini par $F(x, y, z, a, b)=G(x, y, z, a, b)=0$ est lisse de dimension $2 \gamma+1$ et admet comme système de coordonnées 


\section{DÉFORMATIONS ÉQUISINGULIÈRES}

$\left(x, y, z, a_{1}, \ldots, a_{\gamma-1}, b_{1}, \ldots, b_{\gamma-1}\right)$. Notons $\Phi$ la restriction à $x$ de la projection de $\mathbb{c}^{3} \times \mathbb{c}^{2 \gamma}$ sur $\mathbb{C}^{2} \times c^{2 \gamma}: \Phi(x, y, z, a, b)=(x, y, a, b)$.

Un calcul facile montre que le germe de $\Phi$ en 0 est infinitésimalement stable, donc stable. On en déduit ( $\left[\mathrm{Ma}_{2}\right]$ et $\left[\mathrm{B}_{0}\right]$ th.2.6) que $\Sigma^{1}=\left\{M \in X / \operatorname{dim}\left(\operatorname{Ker} T_{M} \Phi\right) \geq 1\right\}$ est un sous espace de codimension au moins égale à 2 ; prenons maintenant un point $P$ de $\Phi(X)-\Phi\left(\Sigma^{1}\right)$ et notons $\Phi^{-1}(p)=\left\{M_{1}, \ldots, M_{k}\right\}$; la stabilité de $\Phi$ implique que les images des espaces tangents à $X$ en $M_{1}, \ldots, M_{k}$ par l'application tangente à $\Phi$ sont en position générale dans $T_{p}\left(\mathbb{C}^{2} \times \mathbb{C}^{2 \gamma}\right)$ :

$$
\operatorname{codim}\left(T_{M_{1}} \Phi\left(T_{M_{1}} X\right) \cap \ldots \cap T_{M_{k}} \Phi\left(T_{M_{k}} X\right)\right)=k
$$

(pour P assez voisin de 0 ).

Il en résulte que 1 'ensemble $Z$ des points du lieu singulier sing $\Phi(X)$ de $\Phi(X)$ oũ $\Phi(X)$ n'est pas isomorphe à l'union de deux sous variétés transverses est de codimension au moins égale à 2 dans $\Phi(X)$. Notons $p$ la restriction à Sing $(\Phi(X))$ de la projection de $\mathbb{c}^{2} x \mathbb{C}^{2 \gamma}$ sur $\mathbb{C}^{2 \gamma} ; p$ est finie par hypothèse. D'après le théorème de Bertini-Sard ( $\left[T_{1}\right]$ page 591 ), $l$ 'image du lieu critique de $p$ est un sous espace strict $S$ de $\mathbb{C}^{2 \gamma}$; donc pour tout $u \in \mathbb{C}^{2 \gamma}-s-p(Z), \pi_{d}\left(X_{u}\right)=\Phi(X) \cap \mathbb{C}^{2} \times\{u\} n^{\prime}$ a comme singularité que des points doubles ordinaires.

\section{PROPOSITION IV.12 :}

Soit $x_{0}$ une courbe intersection complète de $a^{3}$ définie par les équations $f(x, y, z)=g(x, y, z)=0$; on suppose que la direction $d$ de l'axe des $z$ est telle que la projection $\pi_{d}\left(x_{0}\right)$ parallèlement à $d$ de $x_{0}$ est à singularité isolée.

Alors :

$$
2 \iota_{d}=e\left(f\left(x, y, z_{1}\right), g\left(x, y, z_{1}\right), \frac{f\left(x, y, z_{2}\right)-f\left(x, y, z_{1}\right)}{z_{2}-z_{1}}, \frac{\left.g\left(x, y, z_{2}\right)-g\left(x, y, z_{1}\right)\right)}{z_{2}-z_{1}}\right)
$$

multiplicité calculée dans $\mathbb{C}\left\{x, y, z_{1}, z_{2}\right\}$. 
PREUVE :

Soit $p: X \rightarrow U$ une déformation de $X_{0}$ fournie par la proposition IV.11, $X \subset \mathbb{C} \times \mathbb{C}^{3}$ étant défini par les équations $F(u, x, y, z)=G(u, x, y, z)=0$ (au voisinage de 0$)$. Notons $J l$ 'idéal de $\boldsymbol{\sigma}\left\{u, x, y, z_{1}, z_{2}\right\}$ engendré par les fonctions $\left(F\left(u, x, y, z_{1}\right), G\left(u, x, y, z_{1}\right), \frac{F\left(u, x, y, z_{2}\right)-F\left(u, x, y, z_{1}\right)}{z_{2}-z_{1}}, \frac{G\left(u, x, y, z_{2}\right)-G\left(u, x, y, z_{1}\right)}{z_{2}-z_{1}}\right)$

$J$ dēfinit un germe d'espace analytique $K$ fini sur $\mathbb{C} \times\{0\}$ par la projection canonique $P^{\prime}$. Soit $P=\left(x_{0}, y_{0}, z_{1,0}, z_{2,0}\right)$ un point de la fibre $k_{u}=p^{\prime-1}(u)$ où $u$ est fixé non nul assez petit. Nous ne pouvons avoir $z_{1,0}=z_{2,0}$ car sinon, en posant $z_{0}=z_{1,0}=z_{2,0}, F\left(u, x_{0}, y_{0}, z_{0}\right)=G\left(u, x_{0}, y_{0}, z_{0}\right)=$ $F_{z}^{\prime}\left(u, x_{0}, y_{0}, z_{0}\right)=G_{z}^{\prime}\left(u, x_{0}, y_{0}, z_{0}\right)=0$ et la fibre $x_{u}$ aurait au point $\left(x_{0}, y_{0}, z_{0}\right)$ une tangente parallèle à l'axe dès $z$; sa projection aurait alors au point $\left(x_{0}, y_{0}\right)$ une composante irréductible singulière ce qui est impossible par hypothèse. Par conséquent $z_{1,0} \neq z_{2,0}$ et, au voisinage de $P, I$ idéal $J_{u}$ est engendré par les fonctions $\left(F\left(u, x, y, z_{1}\right), G\left(u, x, y, z_{2}\right), F\left(u, x, y, z_{2}\right), G\left(u, x, y, z_{2}\right)\right)$; de plus, puisque le point $\left(x_{0}, y_{0}\right)$ est un point double ordinaire de la projection de $x_{u}$, Tes tangentes à $x_{u}$ en $\left(x_{0}, y_{0}, z_{1,0}\right)$ et $\left(x_{0}, y_{0}, z_{2}, 0\right)$ ne sont pas dans un même plan parallèle à l'axe des $z$; 11 en résulte que les plans tangents aux quatre hy:ersurfaces $F\left(u, x, y, z_{1}\right)=F\left(u, x, y, z_{2}\right)=G\left(u, x, y, z_{1}\right)=G\left(u, x, y, z_{2}\right)=0$ au point $P$ de $C^{4}$ sont transverses et la multiplicité de $J_{u}$ en $P$ est égale à 1 .

Au dessus d'un point double $\left(x_{0}, y_{0}\right)$ de $\pi_{d}\left(x_{u}\right)$ se trouvent exactement deux points $\left(x_{0}, y_{0}, z_{1,0}\right)$ et $\left(x_{0}, y_{0}, z_{2}, 0\right)$ de $x_{u}$, donc deux points $\left(x_{0}, y_{0}, z_{1,0}, z_{2,0}\right)$ et $\left(x_{0}, y_{0}, z_{2,0}, z_{1,0}\right)$ de $k_{u}$; on a donc $e\left(J_{u}\right)=e\left(J_{0}\right)=\sum_{p \in K_{u}} e_{p}\left(J_{u}\right)=2 \ell_{d}$ puisque $K$ est une intersection complète. 


\section{CHAP, $V$ : EXEMPLES}

Avant de donner la liste des exemples, nous allons rappeler sans démonstration une série de résultats qui nous ont été très utiles dans nos calculs.

A. Déformation de la paramètrisation d'une branche :

Nous avons vu (théorème II.3) le résultat de B. Teissier sur la paramètrisation en famille; dans le cas irréductible, R.0. Buchweiss nous en a communiqué la forme suivante :

soient $\left(p_{1}(u, t), \ldots, p_{N}(u, t)\right) N$ fonctions analytiques au voisinage de 0 dans $\mathbb{d}^{2}, s^{\prime}$ annulant en $0, s=\mathbb{C}\left\{p_{1}(u, t), \ldots, p_{N}(u, t)\right\} \subset \mathbb{C}\{u, t\}$, et $S_{0}=\mathbb{C}\left\{p_{1}(0, t), \ldots, p_{N}(0, t)\right\} \subset \mathbb{C}\{t\} \quad ;$ on suppose que $S_{0}$ est l'algèbre locale d'une courbe réduite, c'est à dire que $\delta_{0}=\operatorname{dim}_{\mathfrak{C}}\left(\mathbb{C}\{t\} / S_{0}\right)$ est fini. Les propositions suivantes sont équivalentes.

(i) le morphisme canonique de $S / U S$ dans $S_{0}$ est un isomorphisme.

(ii) le morphisme canonique de $S / U S$ dans $\mathbb{C}\{t\}$ est injectif.

(iii) le $\mathbb{a}\{u\}$-module $\mathbb{d}\{u, t\} / S$ est libre.

Lorsque ces conditions sont satisfaites, l'image de $\mathbb{c}^{2}$ par le morphisme $\left(p_{1}, \ldots, p_{N}\right): \mathbb{C}^{2} \rightarrow \mathbb{C}^{N}$ est un germe de surface $x d^{\prime}$ 'algèbre $\mathcal{O}_{X}=S ; X$ est une déformation plate et à " $\delta$-constant" de sa fibre $X_{0}$ d'algèbre $\mathcal{O}_{X_{0}}=S_{0}$.

B. Caractérisation des branches irréductibles Gorenstein.

Etant donnés un germe de courbe irréductible $X_{0}, \mathcal{O}_{X_{0}} \hookrightarrow \bar{O}_{X_{0}}=\mathbb{C}\{t\}$ sa normalisation, on note $\Gamma\left(X_{0}\right)$ son semi groupe, $c^{\prime}$ est à dire le semi groupe des valuations des 
éléments de ${ }^{X_{0}}$ dans $c\{t\} ; i 1$ existe un plus petit entier $c\left(X_{0}\right)$ (le "conducteur") tel que $c\left(X_{0}\right)+\mathbb{N} \subset \Gamma\left(X_{0}\right)$ et $\delta\left(X_{0}\right)=\operatorname{dim}_{\mathbb{C}}\left(\overline{\mathcal{O}}_{X_{0}} / \mathcal{O}_{X_{0}}\right)=\#\left(\mathbb{N}-\Gamma\left(X_{0}\right)\right)$. Nous dirons que $x_{0}$ est Gorenstein si, pour tout paramètre $x$ de l'anneau local $\mathcal{O}_{X_{0}}, 1$ 'annulateur de 1 'idéal maximal de $\mathcal{O}_{X_{0}} / x^{\mathfrak{O}_{X_{0}}}$ est un $\mathbb{C}$-espace vectoriel de dimension 1. Pour d'autres définitions équivalentes,' voir Kunz [K] ; dans ce même article Kunz démontre l'équivalence des propositions :

(i) $x_{0}$ est Gorenstein

(ii) Le semi groupe $\Gamma\left(X_{0}\right) \subset \mathbb{Z}$ est symétrique : $n \in \Gamma\left(X_{0}\right) \Leftrightarrow c-1-n \notin \Gamma\left(X_{0}\right)$ (iii) $c\left(X_{0}\right)=2 \delta\left(X_{0}\right)$.

On sait bien que si $x_{0}$ est intersection complète, $X_{0}$ est Gorenstein. D'autre part, d'après J.P. Serre ([Se] Prop.5) les notions "Gorenstein" et "intersection complète" sont équivalentes pour les courbes $x_{0}$ dans $\mathbb{c}^{3}$.

\section{Caractérisation des branches monomiales intersections complètes.}

Si on s'occupe des branches monomiales: $\left.\mathcal{O}_{X_{0}}=\mathbb{C}_{\left\{t^{a}\right.}{ }^{a}, t^{a_{2}}, \ldots, t^{a}{ }^{\prime}\right\} c^{\prime}$ est à dire quasi homogène (voir par exemple Pinkham [P] et Buchweiss [Bu] ), on dispose d'un critère réccursif pour déterminer les intersections complètes, donné par C. Delorme dans $[D]$; un semi-groupe $\Gamma$ engendré par un système minimal de générateurs $\left(a_{1}, \ldots, a_{N}\right)$ est d'intersection complète si et seulement si il existe une partition de $\left(a_{1} \ldots a_{N}\right)$ en deux parties $\left(b_{1} \ldots b_{p}\right)$ et $\left(c_{1}, \ldots, c_{N-p}\right)$ telle que :

a) $\left.\left(b_{1}, \ldots, b_{p}\right)=\beta_{1}^{\prime} b_{1}^{\prime}, \ldots, b_{p}^{\prime}\right)$ où $\left(b_{1}^{\prime}, \ldots, b_{p}^{\prime}\right)$ sont premiers entre eux et engendrent un semi groupe $\Gamma^{\prime} d^{\prime}$ intersection complète.

b) $\left(c_{1}, \ldots, c_{N-p}\right)=\gamma\left(c_{1}^{\prime \prime}, \ldots, c_{N-p}^{\prime \prime}\right)$ où $\left(c_{1}^{\prime \prime}, \ldots, c_{N-p}^{\prime \prime}\right)$ sont premiers entre eux et engendrent un semi groupe $\Gamma^{\prime \prime} d^{\prime}$ intersection complète.

c) $\beta$ et $\gamma$ sont premiers entre eux, $B \in \Gamma^{\prime \prime}, \gamma \in \Gamma^{\prime}, B \notin\left\{c_{1}^{\prime \prime}, \ldots, c_{N-p}^{\prime \prime}\right\}$ et $\gamma \notin\left\{b_{1}^{\prime}, \ldots, b_{p}^{\prime}\right\}$. 
Ce critère peut également servir dans le cas où la branche $X_{0} n$ 'est pas monomiale, mais où la courbe monomiale $X_{0}^{\Gamma}$ associée au semi groupe $\Gamma$ de $x_{0}$ est intersection complète ; en effet $x_{0}$ est déformation de la courbe monomiale $x_{0}^{\Gamma}$ ( $\left[T_{3}\right]$ théorème 1.3) et est donc elle aussi intersection complète.

\section{Calcul du nombre de Milnor dans le cas intersections complètes} quasi-homogènes.

Cette formule, d'abord donnée par M. Giusti dans un cas particulier ( $\left[G_{1} i_{1}\right.$ ) puis généralisée par Greuel et Hamm au cas des intersections complètes quasi homogènes de dimension quelconque $\left.\left(\left[{ }_{i}-H\right]\right]\right)$ est la suivante, pour les courbes : soient $\left(w_{1}, \ldots, w_{N}\right)$ les poids des variables $\left(x_{1}, \ldots, x_{N}\right)$, et $\left(d_{1}, \ldots, d_{N-1}\right)$ les poids des polynomes quasi homogènes $\left(f_{1}, \ldots, f_{N-1}\right)$ définissant $x_{0}$; on a : $\mu\left(X_{0}\right)=1+\frac{d_{1} d_{2} \ldots d_{N-1}}{w_{1} w_{2} \ldots w_{N}}\left[\left(d_{1}+d_{2}+\ldots+d_{N-1}\right) \cdots\left(w_{1}+w_{2} \ldots+w_{N}\right)\right]$

EXEMPLE V.1. (J.P. G. Henry):

où $\mu$ est constant, la multiplicité $m$ varie, avec $x_{0}$ intersection complète. On définit dans $\mathbb{C} \times \mathbb{a}^{3}$ la surface $X$ par les deux équations:

$$
\left\{\begin{array}{l}
u z+x y=0 \\
z^{6}+y^{10}+x^{15}=0
\end{array}\right.
$$

$x$ est une intersection complète, donc déformation plate de sa fibre $X_{0}$ définie par les deux équations $x y=0$ et $z^{6}+y^{10}+x^{15}=0$ dans $c^{3}$.

Pour tout $u$ fixé, la fibre $x_{u}$ est quasi homogène : on accorde à $(x, y, z)$ les poids respectifs $(2,3,5)$ et les équations sont alors de degré 5 et 30 ; donc, d'après $D$, le nombre de Milnor $\mu\left(X_{u}\right)$ est constant égal à 126.

Pour $u=0, x_{0}$ a 5 composantes irréductibles : 
$\left\{\begin{array}{c}x=0 \\ z^{3}+i y^{5}=0\end{array} \quad\left\{\begin{array}{l}x=0 \\ z^{3}-i y^{5}=0\end{array} \quad\left\{\begin{array}{l}y=0 \\ z^{2}+x^{5}=0\end{array} \quad\left\{\begin{array}{l}y=0 \\ z^{2}+j x^{5}=0\end{array} \quad\left\{\begin{array}{l}y=0 \\ z^{2}+j^{2} x^{5}=0\end{array}\right.\right.\right.\right.\right.$

et sa multiplicité est égale à 12 .

Pour $u \neq 0, x_{u}$ est isomorphe à une courbe plane (contenue dans. $z=-\frac{x y}{u}$ ) ; $x_{u}$ a 5 composantes irréductibles isomorphes à des cusps (qui ont pour équations $z=-\frac{x y}{u}$ et $y^{2}-\varepsilon_{i} x^{3}=0$ où $\varepsilon_{i}$ pour $i=1,2 \ldots 5$ parcourt les 5 racines de l'équation: $\varepsilon^{5}+\frac{\varepsilon^{3}}{u^{6}}+1=0$ ).

Donc $x_{u}$ est de multiplicité 10 .

Décrivons plus précisément $l$ 'une des composantes irréductibles de $X$ par exemple $x^{3}$ image directe de l'application $\varphi:\left(\mathbb{C}^{2}, 0\right) \rightarrow\left(\mathbb{C} \times \mathbb{d}^{3}, 0\right)$

définie par :

$\varphi(t, u)=\left[u, x(t, u)=-t^{2}, y(t, u)=u \beta(u) t^{3}, z(t, u)=\beta(u) t^{5}\right]$

où $\beta(u)$ est la racine de $1^{\text {'équation }} \beta^{6}+u^{10} \beta^{10}-1$ qui vaut 1 pour $u=0$ (donnée par le théorème des fonctions implicites).

L'idéal premier définissant $x^{3}$ est le noyau de $\varphi^{*}: \mathbb{C}\{x, y, z, u\} \rightarrow \mathbb{C}\{t, u\}$ dont un système de générateur est :

$I^{3}=\left(x y+u z, y^{2}+u^{2} \beta^{2} x^{3}, z^{2}+\beta^{2} x^{5}, z y-u \beta^{2} x^{4}\right)$

on constate que la fibre en 0 de $x^{3},\left(x^{3}\right)_{0}$ est définie dans $\left(C^{3}, 0\right)$ par l'içáal non réduit :

$I_{0}^{3}=\left(x y, y^{2}, z^{2}+x^{5}, z y\right)=\left(y, z^{2}+x^{5}\right) n\left(x, y^{2}, z\right)$

et $\left(x^{3}\right)_{0}$ a une composante immergée à l'origine. Il ne pouvait en être autrement d'après la proposition II.7.

\section{EXEMPLE V.2.:}

où $\mu$ est constant, la multiplicité $m$ varie, avec $X_{0}$ irréductible, Gorenstein.

On se donne la sous algèbre $S$ de $a\{u, t\}$ engendrée par: 
$\left(x_{1}(t, u)=t^{6}+u t^{5}, x_{2}(t, u)=t^{10}, x_{3}(t, u)=t^{11}, x_{4}(t, u)=t^{14}-u t^{13}, x_{5}(t, u)-t^{15}\right)$. Pour $u=0, l^{\prime}$ algèbre $S_{0}=c\left\{t^{6}, t^{10}, t^{11}, t^{14}, t^{15}\right\} \subset \mathbb{C}\{t\}$ est monomiale, de semi groupe $\Gamma\left(X_{0}\right)$ :

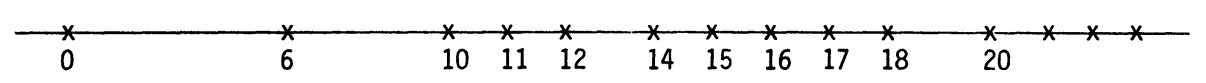

Ce semi-groupe est symétrique, $c=2 \delta\left(X_{0}\right)=20$, et donc $X_{0}$ est une branche Gorenstein dans $\mathbb{C}^{5}$ (d'après B.)

Pour $u$ fixé non nul: l'algèbre $S_{u}=\mathbb{C}\left\{t^{6}+u t^{5}, t^{10}, t^{11}, t^{14}-u t^{13}, t^{15}\right\} \subset \mathbb{C}\{t\}$

a pour semi groupe de valuations :

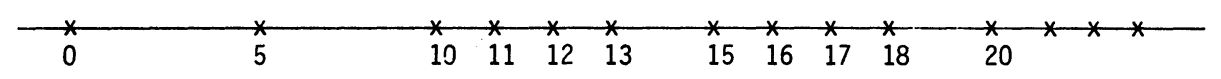

On a encore $c=2 \delta\left(X_{u}\right)=20$. Par conséquent, d'après $A$., la surface $X$ de $c^{5} \times C$ d'algèbre $S$ est une déformation plate de sa fibre $x_{0} \check{\alpha}^{\prime \prime} \mu=2 \delta$ constant."

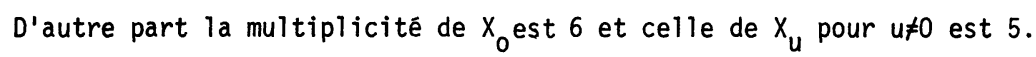

EXEMPLE V.3. :

où le semi groupe $\Gamma$ est constant, où il n'y a pas équisaturation, avec $x_{0}$ irréductible intersection complète.

Soit $S=\mathbb{a}\left\{t^{4}, t^{6}+u t^{7}, t^{9}\right\} \subset \mathbb{C}\{u, t\}$; pour tout $u$ fixé, l'algèbre $S_{u}=\mathbb{a}\left\{t^{4}, t^{6}+u t^{7}, t^{9}\right\} \subset \mathbb{a}\{t\}$ a pour semi groupe de valuations :

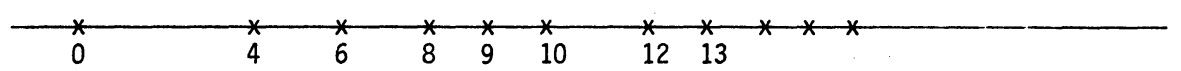

Ce semi groupe $\Gamma$ est constant, $c=2 \delta=12$; et donc, d'après $A$, la surface $X$ de $a^{3} x \mathbb{C} d^{\prime}$ algębre $S$ est une déformation à semi-groupe constant de sa fibre $x_{0} d^{\prime}$ algèbre $S_{0}=C\left\{t^{4}, t^{6}, t^{9}\right\} \subset \mathbb{a}\{t\}$ qui est une intersection complète. Par ailleurs 1 'algèbre $S_{0}$ saturée de $S_{0}$ est $\mathbb{C}\left\{t^{4}, t^{6}, t^{9}, t^{11}\right\}$, alors que, pour $u \neq 0, l^{\prime}$ algèbre $\hat{S}_{u}$ saturée de $S_{u}$ est $\mathbb{a}\left\{t^{4}, t^{6}, t^{7}, t^{9}\right\}$. Autrement dit (théorème IV.8). 
la famille des projections planes génériques $\left(X=t^{4}, Y=t^{6}+u t^{7}+\lambda t^{9}\right) n^{1}$ est pas équisingulière (pour $u=0$ la courbe a deux paires de Puiseux $(2,3)$ et $(2,9)$ tandis que pour $u \neq 0$ les deux paires de Puiseux sont $(2,3)$ et $(2,7))$. Il n'y a donc pas équisaturation.

EXEMPLE V.4. :

oũ il y a équisaturation, oũ le semi groupe $n$ 'est pas constant, avec $x_{0}$ irréductible, Gorenstein.

$S=c\left\{t^{9}, t^{11}, t^{16}+u t^{14}, t^{21}-u t^{19}, t^{23}, t^{28}\right\} \subset a\{u, t\}$

Pour $u=0$, le semi-groupe des valuations de $S_{0} \subset \mathbb{C}\{t\}$ est :

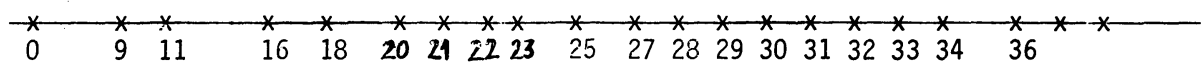

$S_{0}$ est Gorenstein avec $c=2 \delta=36$.

Pour u fixé non nul, le semi-groupe des valuations de $S_{u} \subset \mathbb{C}\{i\}$ est :

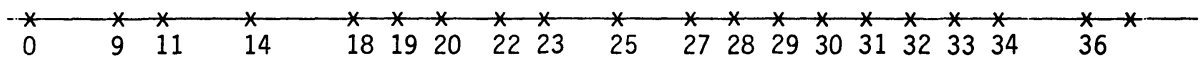

$S_{u}$ est encore Gorenstein avec $c=2 \delta=36$, mais le semi groupe $n$ 'est pas le même que pour $u=0$.

D'autre part, puisque 9 et 11 sont premiers entre eux, $S_{0}$ et $S_{u}$ ont même saturé que $\mathbb{C}\left\{t^{9}, t^{11}\right\} \subset \mathbb{C}\{t\}$ et $i 1$ y a donc équisaturation.

\section{REMARQUE :}

Si on n'exige pas $X_{0}$ Gorenstein, on a des exemples beaucoup plus simples avec équisaturation et semi-groupe non constant :

$s=\mathbb{C}\left\{t^{5}, t^{6}, t^{8}+u t^{7}\right\} \subset \mathbb{C}\{u, t\}$.

EXEMPLE V.5.:

où les conditions de Whitney sont satisfaites, mais où le discriminant de la projection sur un plan générique passant par l'axe des u n'est pas équimultiple. 
On considère la sous algèbre $S=\mathbb{C}\left\{t^{4}, t^{6}+u t^{7}, t^{11}, t^{13}+\frac{u}{2} t^{14}\right\}$

de $C\{u, t\}$; pour $u=0, S_{0}=\mathbb{C}\left\{t^{4}, t^{6}, t^{11}, t^{13}\right\}$ est $l^{\prime}$ algèbre de la courbe quasi homogẽne de $\mathbb{C}^{4}$ d'équations :

$\omega^{2}-x^{5} y=z \omega-x^{6}=y \omega-x^{2} z=x \omega-y z=z^{2}-x^{4} y=y^{2}-x^{3}=0$

Son nombre de Milnor est $\mu\left(X_{0}\right)=2 \delta\left(X_{0}\right)=12$.

On note I l'idéal de $\mathbf{C}\{x, y, z, \omega\}$ définissant $x_{0}$, J l'idéal définissant dans $x_{0}$ le lieu critique de la projection de $x_{0}$ sur l'axe des $x$; J est engendré par les mineurs $3 \times 3$ de la matrice :

$$
\left(\begin{array}{cccccc}
-x^{5} & 0 & \omega & -z & -x^{4} & 2 y \\
0 & \omega & -x^{2} & -y & 2 z & 0 \\
20 & z & y & x & 0 & 0
\end{array}\right)
$$

On trouve que $\mathrm{J}$ est l'idéal de $S_{0}$ engendré par les monomes $t^{21}, t^{23}, t^{28}, t^{30}$ et que la colongueur de $J$ dans $S_{0}$ est égale à $\Delta_{0}=18$. C'est la multiplicité du discriminant de la projection de $x_{0}$ sur l'axe des $x:$ on constate que $\Delta_{0}>\mu\left(X_{0}\right)+m\left(X_{0}\right)-1=15$.

Pour $u \neq 0$ on a encore $\mu\left(X_{u}\right)=12$. Et on trouve que la multiplicité du discriminant de la projection de $X_{u}$ sur l'axe des $x$ est $\Delta_{u}=15=\mu\left(X_{u}\right)+m\left(X_{u}\right)-1$ $S$ est 1 'algèbre d'une surface $X$ de $C^{4} x C$ déformation plate de sa fibre, $(X-U, U)$ vérifie les conditíons de Whitney (puisque $\mu$ et $m$ sont constants) et le discriminant de la projection de $X$ sur le plan des variables $(u, x) n$ 'est pas principal (il a une composante immergée en 0 ) bien que le lieu discriminant réduit soit identique à l'axe des $u$.

REMARQUES : R.0. Buchweiss nous a indiqué un autre exemple avec en plus $x_{0}$ Gorenstein : $S=C\left\{t^{6}, t^{8}+2 u t^{9}, t^{10}+u t^{11}, t^{17}, t^{19}+\frac{2 u}{3} t^{21}\right\}$. 
Ici $\mu+m-1=27$ est constant, et la multiplicité du discriminant de la projection générique est 33 pour $u=0$ et 27 pour $u \neq 0$ (car $S_{u}$ est alors intersection complète).

Dans tous les exemples de courbes monomiales $x_{0}$ de $c^{3} x=t^{a}, y=t^{b}, z=t^{c} a<b<c$ que nous avons calculés nous avons trouvé : $\Delta_{0}=\mu\left(X_{0}\right)+m\left(X_{0}\right)-1 . \cdots$

\section{EXEMPLE V.6. :}

où la strate à $\mu$-constant $n$ 'est pas lisse, avec $X_{0}$ irréductible dans $\mathbf{c}^{3}$. Nous allons voir que dans l'exemple le plus simple possible de branche irréductible non intersection complète de $c^{3}$ (d'algèbre $S_{0}=c\left(t^{3}, t^{4}, t^{5}\right\}$ ), la strate à $\mu$-constant dans la déformation mini-verselle $n$ 'est pas lisse.

M. Schaps a montré ( $[S c]$ Théorème 1), qu'une courbe réduite $x_{0}$ de $c^{3}$ est déterminantielle et que la base de sa déformation miniverselle est lisse ; elle donne l'exemple de la courbe $x_{0} d^{\prime}$ 'algèbre $S_{0}=c\left(t^{3}, t^{4}, t^{5}\right\}$ et montre que sa déformation miniverselle est donnée par la restriction à $Z \subset C^{3} \times C^{5}$ de la projection canonique de $\mathbf{c}^{3} \times \mathbb{C}^{5}$ sur $\mathbb{C}^{5}, Z$ étant défini par 1 'annulation des mineurs d'ordre 2 de la matrice :

$$
R=\left(\begin{array}{lll}
z & x^{2}+x T_{1}+T_{2} \\
y & z+x T_{3}+T_{4} \\
x & y+T_{5}
\end{array}\right)
$$

Soit $x \subset \mathbb{C}^{3} \times \mathbb{C}$ une déformation à un paramètre à $\mu$-constant de $x_{0}$-déformation à priori non centrée - et qu'on suppose non triviale.

Puisqu'ici une déformation de $x_{0}$ à semi-groupe constant est triviale, pour $u$ fixé non nul l'algèbre $S_{u}$ de la fibre $x_{u}$ en son point singulier est isomorphe à $c\left\{t^{2}, t^{5}\right\}$. 


\section{DÉFORMATIONS ÉQUISINGULIÈRES}

Par définition de la déformation mini-verselle, il existe un carré cartésien :

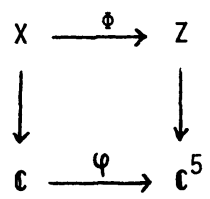

(et l'application linéaire tangente au changement de base $\varphi$ est unique).

En composant $\Phi$ et la paramètrisation en famille de $x, n: c^{2} \longrightarrow x$, nous obtenons :

$$
\begin{aligned}
& x(t, u)=x_{0}(u)+\sum_{i \geq 2}^{\Sigma} x_{i}(u) t^{i}+t^{3} \\
& y(t, u)=y_{0}(u)+\sum_{i \geq 2}^{\Sigma} y_{j}(u) t^{i}+t^{4} \\
& z(t, u)=z_{0}(u)+\sum_{i \geq 2}^{\Sigma} z_{i}(u) t^{i}+t^{5} \\
& \varphi(u)=\left(T_{1}(u), \ldots, T_{5}(u)\right)
\end{aligned}
$$

ou les fonctions $x_{j}(u), y_{i}(u), z_{i}(u)\left(i=0\right.$ et $i \geq 2$ ) et $T_{1}(u), \ldots, T_{5}(u)$ sont dans l'idéal maximal de $\mathbf{C}\{u\}$ et où la matrice

$$
M(u)=\left(\begin{array}{ccc}
x_{2}(u) & y_{2}(u) & z_{2}(u) \\
1+x_{3}(u) & y_{3}(u) & z_{3}(u)
\end{array}\right) \text { est de rang } 1 .
$$

En écrivant que $\Phi_{\diamond} n(t, u)$ annule les déterminants d'ordre 2 de la matrice $R$, et par identification (nous ne reproduisons pas ici ces calculs fastidieux) on trouve que $T_{1}(u), \ldots, T_{5}(u)$ sont dans le carré de l'idéal maximal de $C\{u\}$ : cela implique que le changement de base $n$ 'est jamais une immersion, et donc que la strate à $\mu$-constant $n$ 'est pas lisse.

\section{REMARQUE :}

On peut aussi montrer que la déformation $x=t^{3}+u t^{2}, y=t^{4}, z=t^{5}$ est "verselle pour les déformations à $\mu$-constant", et que le changement de base $\varphi: C \longrightarrow c^{5}$ correspondant à cette déformation particulière $n$ 'est pas une immersion. 


\section{APPENDice : SATURATION DES ALGËBRES ANALYTIQUES LOCALES DE DIMENSION UN (D'APRÈs F. PHAM ET Bi. TEISSIER)}

\section{$\S 0$. INTRODUCTION :}

Soit $C \subset\left(\mathbb{C}^{N}, 0\right)$ un germe de courbe analytique complexe réduite d'anneau local $A$. On se propose de: montrer comment le critère valuatif de dépendance intégrale $\left(\left[\mathrm{T}_{4}\right]\right)$ permet de déterminer la structure du saturé Lipschitzien $\tilde{A}$ de $A$ au sens de F. Pham et B. Teissier ([P.T.]).

Soit $I_{A}$ le noyau de $l$ 'homomorphisme $\bar{A} \bar{A} \longrightarrow \bar{A} \bar{A}$ où désigne le produit tensoriel analytique et $\bar{A}$ le normalisé de $A$; rappelons la définition du saturé de A :

DEFINITION VI.0.1. ([P.T]) :

Le saturé Lipschitzien de $A$ est le sous anneau $\widetilde{A}$ de $\bar{A}$ formé des $f \in \bar{A}$ tels que $f 1$ - 1 soit dans la clôture intégrale $\bar{I}_{A}$ de $I_{A}$.

Soient $C_{1}, \ldots, C_{r}$ les branches de $C, A_{i} l$ 'anneau local de $C_{i}$; on a $\bar{A} \approx \stackrel{r}{\oplus_{1}} \bar{A}_{i}$; si $x$ est un paramètre transverse de $A\left(\left[Z_{2}\right]\right)$, on peut trouver $t_{j} \in \bar{A}_{j}$ tel que $\bar{A}_{i}=\mathbb{C}\left\{t_{i}\right\}$ (uniformisante de $A_{j}$ ) et on peut supposer que $x=t_{1}^{\nu_{1}} \oplus \ldots \oplus t_{r}^{\nu_{r}}$ grâce à 1 'identification $A \subset \bar{A}=\mathbb{C}\left\{t_{1}\right\} \otimes \ldots \oplus \mathbb{C}\left\{t_{r}\right\} \quad\left(\nu_{j}\right.$ est la multiplicité de $\left.c_{j}\right)$. On appelle $\left(t_{1}, \ldots, t_{r}\right)$ une bonne famille d'uniformisantes et $t_{1}=t$ une bonne uniformisante si $r=1$.

Soit $\left(z_{p}=\bigoplus_{i=1}^{r} z_{p, i} ; p=1, \ldots, N\right)$ un système de générateurs de l'idéal maximal $m$ de $A ; 1$ idéal $I_{A}$ est engendré par les éléments $z_{p} 1-1 z_{p}, p=1, \ldots, N$. Soit $I_{A}^{(i, j)} \quad l$ idéal de $C\left\{t_{j}, t_{j}^{\prime}\right\}$ engendré par les éléments $z_{p, i}\left(t_{j}\right)-z_{p, j}\left(t_{j}^{\prime}\right)$ $p=1, \ldots, N$. 


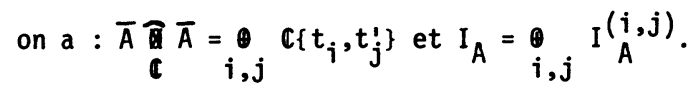

Un èlēment $f=f_{1} \oplus \ldots f_{r}$ de $\bar{A}$ est dans $\widetilde{A}$ si et seulement si pour tout couple $(i, j)$ on $a: f_{i} 1-1 f_{j} \in I_{A}^{(i, j)}$.

D'après le critère valuatif de dépendance intégrale cețte dernière condition équivaut à la condition suivante notée $\left(P_{i, j}\right)$ :

$\left(P_{i, j}\right)$ Pour tout homomorphisme local b $: C\left\{t_{j}, t_{j}^{1}\right\} \longrightarrow C\{u\}$ de valuation associée $v$ :

$$
v\left(f_{i}\left(t_{j}\right)-f_{j}\left(t_{j}^{\prime}\right)\right) \geq v\left(I_{A}^{(i, j)}\right) .
$$

La description du saturé d'une algèbre analytique $A$ aboutit au résultat suivant démontré au $\$ .3$.

\section{THÉORĒME VI.0.2.}

Deux germes $C$ et $D$ de courbes analytiques complexes réduites d'anneaux locaux $A$ et $B$ ont des saturés $\AA^{A}$ et $\widetilde{B}$ isomorphes si ei seulement si leurs projections planes génériques sont topologiquement équivalentes (ou (a)-équivalentes au sens de $\left.\left[Z_{1}\right]\right)$.

Précisément nous montrons que si $A$ est $l$ 'algèbre d'une branche irréductible $C$, son saturé $\tilde{A} \subset \mathbb{C}\{t\}$ est engendré par des monômes déterminés par les exposants caractéristiques de la projection plane générique de C (théorème VI.1.6, proposition VI.3.1 et lemme VI.3.3).

Dans le cas réductible, nous montrons que pour deux branches distinctes $C_{\mathbf{i}}$ et $C_{j}$, la condition $\left(P_{i, j}\right) n^{\prime} a$ à être vérifiée que pour un nombre fini de valuations bien définies, si on sait déjà que $f_{j} \in \widetilde{A}_{j}$ pour $i=1 \ldots r$ (théorème VI.2.2) et même une seưle valuation (lemme VI.3.6). Nous démontrons au passage qu'une fraction Lipschitzienne sur une branche $C_{i}$ se prolonge en une fraction Lipschitzienne sur C (lemme VI.3.7). Ceci nous permet alors de déterminer le saturé $\tilde{A}$ de $A$ en fonction des exposants caractéristiques des branches de la projection plane générique de $C$ et de la multiplicité d'intersection de ces branches deux à deux (proposition VI.3.1 et VI.3.2). Et réciproquement la donnée de $\tilde{A}$ permet de calculer ces invariants numériques. 


\section{$\S$ 1. CAS IRREDUCTIBLE :}

Dans le cas $r=1$, on choisit une bonne uniformisante $t$ de $A$ et on a : $\bar{A}=C\{t\}$, $\bar{A} \hat{B}_{C} \bar{A}=\mathbb{C}\left\{t, t^{\prime}\right\}$. Etant donné $f=\sum_{p \geq 0}^{\sum} a_{p} t^{p \in A}$ on a

$f 1-1 f=\sum_{p \geq 0}^{\Sigma} a_{p}\left(t^{p}-t^{\prime p}\right)$, ce qui nous conduit à étudier les valuations des éléments de la forme $t^{p}-t^{\prime p}$ :

LEMME VI.1.1. :

a) A tout homomorphisme local b : $\mathbb{C}\left\{t, t^{\prime}\right\} \longrightarrow \mathbb{C}\{u\}$ sont associés deux entiers $e \geq 0, v_{0}>0$, et, lorsque e $>0$, un entier $v_{1}>v_{0}$ ou $v_{1}=\infty$ tels que, pour tout $p \in \mathbb{N}^{*}$ on ait :

$$
\begin{aligned}
& v\left(t^{p}-t^{\prime} p\right)=p v_{0} \text { si e ne divise pas } p \\
& v\left(t^{p}-t^{\prime p}\right)=(p-1) v_{0}+v_{1} \text { si e divise } p
\end{aligned}
$$

b) Inversement, étant donnés e, $v_{0}, v_{1}$ satisfaisant les conditions de a), on peut trouver $b$ tel que les valuations des éléments $t^{p}-t^{\prime} p$ soient données par les formules précédentes.

\section{PREUVE :}

a) On note $v_{0}=\inf \left(v(t), v\left(t^{\prime}\right)\right)$ et on distingue deux cas :

- si pour tout $p, v\left(t^{p}-t^{\prime p}\right)=p v_{0}$, on prend $e=0$

- sinon $i l$ existe $\xi$ racine de l'unité d'ordre e telle que $v\left(t^{\prime}-\xi t\right)=v_{1}>v_{0}$ et e, $v_{0}, v_{1}$ satisfont $a$ ).

b) il suffit de prendre $b(t)=u^{v_{0}}, b\left(t^{\prime}\right)=\xi u^{v_{0}}+u^{v_{1}}$ où $\xi$ est une racine de l'unité d'ordre e $>0$, ou $\xi=0$ si $e=0$.

DÉFINITION VI.1.2. :

Sott $f=\sum_{p \geq 0} a_{p} t^{p} \in \bar{A}$. On appelle ensemble des exposants de $f$ l'ensemble d'entiers $\operatorname{Ex}(f)=\left\{p \in \mathbb{N} / a_{p} \neq 0\right\}$. 
On appelle ensemble des exposants de $A=E(A)=\underset{f \in m}{U} E x(f)$.

\section{LEMME VI.1.3. :}

Tout monơme $t^{p}$ tel que $p \in E(A)$ est un élément de $\AA$.

\section{PREUVE :}

Raisonnons par récurrence. Soit $q \in E(A)$ tel que, pour tout $p<q$ tel que $p \in E(A)$, on ait $t^{P} \in \tilde{A}$; soit $A_{1} l$ 'anneau engendré par $A$ et les monômes $t^{p}$ pour $p \in E(A)$, $p<q$. D'après $l$ 'hypothèse de récurrence et le fait que $\widetilde{\widetilde{A}}=\widetilde{A}, \AA_{1}=\tilde{A}$, et il suffit donc de montrer que, pour toute valuation $v$ de $C\left\{t, t^{\prime}\right\}, v\left(t^{q}-t^{\prime q}\right) \geq v\left(I_{A_{1}}\right)$. Distinguons, d'après le lemme VI.1.1, deux cas :

1) si $v\left(t^{q}-t^{\prime} q\right)=q v_{0}, i 1$ existe $f=t^{q}+\sum_{s>q}>a_{s} t^{s} \in A_{1}$.

Donc: $v\left(t^{q}-t^{\prime} q\right)=q v_{0}=v\left(f(t)-\dot{f}^{\prime}\left(t^{\prime}\right)\right) \geq v\left(I_{A_{1}}\right)$.

2) sinoo $v\left(t^{q}-t^{\prime} q\right)=(q-1) v_{0}+v_{1} \geq(v-1) v_{0}+v_{1} \geq v\left(t^{v}-t^{\prime v}\right) \geq v\left(I_{A_{1}}\right)$

où $\nu$ est la multiplicité de $A\left(\right.$ et $\left.t^{\nu} \in A \subset A_{1}\right)$.

COROLLAIRE VI.1.4. :

$L$ 'anneau $A$ est monomial : $\mathscr{A}=\mathbb{C}\left\{t^{p} ; p \in E(\widetilde{A})\right\}$

DÉFINITION VI.1.5. :

Soit $E$ une partie de $\mathbb{N}^{*}$. On note $E_{q}=\{p \in E, p \leq q\}$ et on appelle saturé de $E$ l'ensemble $\tilde{E}=\left\{q \in N^{*} / q\right.$ multiple du pgcd des éléments de $\left.E_{q}\right\}$.

Soit $t^{r} e_{0}=\beta_{0}=\inf \{p \in E\}, \tilde{E}_{0}=E \cup \beta_{0} N^{*}$ et définissons deux suites d'entiers $e_{0}>e_{1}>\ldots>e_{g}=\operatorname{pgcd}\{p / p \in E\}$ et $\beta_{0}<\beta_{1} \ldots<\beta_{g}$, ainsi qu'une suite $\tilde{E}_{0} \subset \ldots \subset \tilde{E}_{g}$ de sous-ensembles de $\mathbb{N}$ par les formules suivantes :

$\beta_{i+1}=\inf \left\{p \in E / e_{i}\right.$ ne divise pas $\left.p\right\}, e_{i+1}=\operatorname{pgcd}\left\{\beta_{0}, \beta_{1} \ldots, \beta_{i+1}\right\}$ et $\tilde{E}_{i+1}=\tilde{E}_{i} \cup\left\{\beta_{i+1}+e_{i+1} \mathbb{N}^{*}\right\}$. 
On a $\tilde{E}=\tilde{E}_{g}=\left\{\beta_{0}, \beta_{1} \ldots, \beta_{g}\right\}^{2}$ et on appelle $\left\{\beta_{0}, \ldots, \beta_{g}\right\}$ la suite caractéristique de $E$ (de $A$ si $E=E(A)$ ).

THÉORËME VI.1.6. :

Soit $A \bar{C} \bar{A}=\mathbb{C}\{t\} l^{\prime}$ 'anneau d'un germe de courbe analytique irréductible muni d'une bonne uniformisante $t$. Le saturé de $A$ est l'anneau monomial

$\tilde{A}=\mathbb{C}\left\{t^{p} / p \in \tilde{E}(A)\right\}$.

Ainsi le saturé de $A$ est déterminé à isomorphisme près par la suite caractéristique de $A$ (qui est aussi celle de $\tilde{E}(A)$ ).

PREUVE :

i1 s'agit de montrer que $\widetilde{E(A)}=E(\AA)$. Soit $v$ une valuation de $\mathbb{C}\left\{t, t^{\prime}\right\}, v_{0}, v_{1}, e$ les entiers associés à $v$ par le lemme $V I .1 .1$ et $i_{e}=\inf \left\{k / e\right.$ ne divise pas $\left.\beta_{k}\right\}$. Remarquons que, d'après le leme VI.1.3: $v\left(I_{A}\right)=\inf \left\{v\left(t^{P}-t^{\prime} P\right) / p \in E(A)\right\}$.

Soit $p \in \hat{E}(A)$ on a :

1) si $p<\beta_{i_{e}}, v\left(t^{p}-t^{\prime p}\right)=(p-1) v_{0}+v_{1} \geq\left(\beta_{0}-1\right) v_{0}+v_{1}=v\left(t^{\beta}-t^{\prime \beta_{0}}\right)$.

2) si $p \geq \beta_{i_{e}}, v\left(t^{p}-t^{\prime p}\right) \geq p v_{0} \geq \beta_{i_{e}} v_{0}=v\left(t^{\beta^{\beta}} i_{e}-t^{{ }^{\beta} i_{e}}\right)$.

Il en résulte que $v\left(t^{p}-t^{\prime} P\right) \geq v\left(I_{A}\right)$, donc $t^{P} \in \tilde{A}$ et $\tilde{E}(\tilde{A}) \subset E(\tilde{A})$.

Pour démontrer l'inclusion inverse, remarquons que ces formules appliquées à tous les $p \in E(A)$ montrent que :

$$
v\left(I_{A}\right)=\inf \left(\beta_{i_{e}} v_{0} ;\left(\beta_{0}-1\right) v_{0}+v_{1}\right)
$$

si $q \notin \widetilde{E(A)}$, il s'agit de montrer que $q \notin E(\tilde{A})$ ou $t^{q} \notin \tilde{A}$ :

soit $i \in\{0, \ldots, g\}$ défini par $\beta_{i-1}<q<\beta_{j}$ (par convention $\beta_{-1}=0$ ); on note $e=e_{i-1}$ si $i \geq 1$, e=0 si $i=0$ et on remarque que $i=i$ et $e$ ne divise pas $q$.

$D^{\prime}$ après le lemme VI.1.1 il existe une valuation $v$ de $\mathbb{C}\left\{t, t^{\prime}\right\}$ telle que :

$$
\begin{aligned}
& v\left(t^{q}-t^{\prime} q\right)=q v_{0}<\beta_{i_{e}} v_{0}<\left(\beta_{0}-1\right) v_{0}+v_{1} \\
& \text { donc } v\left(t^{q}-t^{\prime} q\right)<v\left(I_{A}\right) \text { et } t^{q} \notin A .
\end{aligned}
$$




\section{DÉFORMATIONS ÉQUISINGULIÈRES}

\section{$\S 2$. CAS RÉDUCTIBLE :}

Choisissons une bonne famille d'uniformisantes $\left(t_{1}, \ldots, t_{r}\right)$ de $A$, et

$\mu=\mu_{i} v_{j}(1 \leq i \leq r)$ un multiple commun à toutes les multiplicités. Pour toute branche $C_{i}$, définissons 1 'homomorphisme local $\varphi_{i}: \bar{A}_{i}=\boldsymbol{C}\left\{t_{j}\right\} \longrightarrow c\{\tau\} \varphi_{i}\left(t_{i}\right)=\tau^{\mu_{i}}$. Soient $C_{\mathbf{i}}$ et $C_{\mathbf{j}}$ deux branches distinctes :

DÉFINITION VI.2.1. :

pour toute racine $\mu$-ième de l'unité, $\varepsilon$, et tout $f=f_{1} \oplus \ldots \oplus f_{r} \in \bar{A}$, nous appelons $\varepsilon$-valuation de $f$ le nombre

$m_{i, j, \varepsilon}(f)=v_{\tau}\left[\varphi_{i}\left(f_{i}\right)(\tau)-\varphi_{j}\left(f_{j}\right)(\varepsilon \tau)\right]=v_{\tau}\left[f_{j}\left(\tau^{{ }^{\mu}}{ }^{i}\right)-f_{j}\left((\varepsilon \tau)^{{ }^{\mu} j}\right)\right]$

et $\varepsilon$-valuation de $A$ le nombre $m_{i, j, \varepsilon}=\inf \left\{m_{i, j, \varepsilon}(f) ; f \in A\right\}$.

\section{THEEORẼME VI.2.2:}

Soit $\left.A \subset \bar{A}=\mathbb{C}\left\{t_{1}\right\} \otimes \ldots \otimes C t_{r}\right\}$ une algèbre analytique réduite de dimension un, munie d'une bonne famille d'uniformisantes $\left(t_{1}, \ldots, t_{r}\right)$. Pour que $f=f_{1} \oplus \ldots \oplus f_{r} \in \bar{A}$ soit un élément de $\AA$, il faut et $i l$ suffit que les deux conditions suivantes soient satisfaites :

(a) Pour tout $i \in\{1, \ldots, r\}, f_{i} \in \hat{A}_{i}$.

(b) Pour tout $(i, j) \in\{1, \ldots, r\}^{2}$, $i \neq j$, et tout $\varepsilon$ racine $\mu$-ième de l'unité

$$
m_{i, j, \varepsilon}(f) \geq m_{i, j, \varepsilon} \text {. }
$$

REMARQUE :

Nous verrons (lemme VI.3.6) après l'étude du saturé des germes de courbes planes que cette dernière condition peut être remplacée par la seule condition suivante pour $(i, j)$ fixé et $\underline{\varepsilon}$ racine $\mu$-ème de l'unité telle que

$m_{i, j, \underline{\varepsilon}}=\sup \left\{m_{i, j, \varepsilon} ; \varepsilon^{\mu}=1\right\}:\left(b^{\prime}\right) m_{i, j, \underline{\varepsilon}}(f) \geq m_{i, j, \underline{\varepsilon}}$.

D'autre part, il résulte de (a) et du théorème VI.1.6 que $A$ et $\tilde{A}$ ont même multiplicité. 
PREUVE DU THÉORËME VI.2.2.

La condition $\left(P_{i, j}\right)$ de 1 'introduction équivaut à $f_{i} \in \mathscr{A}_{i} \operatorname{car} \underset{A}{(i, i)}=I_{A_{j}}$ et la condition (a) du théorème est donc nécessaire.

Pour deux entiers $(i, j)$ distincts parmi $\{1, \ldots, r\}$ et $v$ une valuation de

$\left.\mathbf{C}_{\{} t_{i}, t_{j}^{\prime}\right\}$ définie par 1 'homomorphisme local $b: \mathbb{C}\left\{t_{i}, t_{j}^{1}\right\} \rightarrow \mathbb{C}\{u\}$, distinguons deux cas :

ler Cas :

$v\left(t_{i}^{\nu_{i}}-t_{j}^{\nu_{j}}\right)=\inf \left(v\left(t_{i}^{\nu_{i}}\right), v\left(t_{j}^{\nu_{j}}\right)\right)=v\left(I_{A}^{(i, j)}\right)$;

comme $A_{i}$ et $\tilde{A}_{i}$ (resp. $A_{j}$ et $\tilde{A}_{j}$ ) ont la même multiplicité $\nu_{i}\left(\right.$ resp. $\left.\nu_{j}\right)$,

la condition $\left(P_{i, j}\right)$ pour une telle valuation équivaut à $f_{j}(0)=f_{j}(0)$.

\section{2ème Cas :}

$v\left(t_{i}^{\nu_{i}}-t_{j}^{\nu_{j}}\right)>\inf \left(v\left(t_{i}^{\nu_{i}}\right), v\left(t_{j}^{\nu_{j}}\right)\right)$.

Cette condition implique $\nu_{i} v_{i}=\nu_{j} v_{j}$ oũ $v_{i}=v\left(t_{i}\right)$ et $v_{j}=v\left(t_{j}\right)$.

Notons $\psi: \mathbb{C}\left\{t_{i}, t_{j}^{\prime}\right\} \longrightarrow \mathbb{C}\left\{\tau, \tau^{\prime}\right\} \quad l$ 'homomorphisme défini par $\psi\left(t_{i}\right)=\tau^{\mu_{i}}$,

$\psi\left(t_{j}^{\prime}\right)=\tau^{\prime{ }^{\mu} j}$; E et $F$ les unités de $\mathbb{C}\{u\}$ telles que $b\left(t_{i}\right)=u^{v_{i}}$ et $b\left(t_{j}^{\prime}\right)=u^{v^{j_{F}}}$.

Nous pouvons choisir alors un homomorphisme $\tilde{b}: \mathbb{C}\left\{\tau, \tau^{\prime}\right\} \longrightarrow \mathbb{C}\{u\}:$

$\tilde{b}(\tau)=u^{\nu_{i} v_{i}}\left[E\left(u^{\mu}\right)\right]^{1 / \mu_{i}}$ et $\tilde{b}\left(\tau^{\prime}\right)=u^{\nu_{j} v_{j}}\left[F\left(u^{\mu}\right)\right]^{1 / \mu_{j}}$.

On vérifie alors :

$\tilde{b}_{\circ \psi}\left(t_{j}\right)=u^{\mu v_{i}} E\left(u^{\mu}\right)=b\left(t_{j}\right)\left(u^{\mu}\right)$

$\tilde{\tilde{b}_{\circ} \psi}\left(t_{j}^{\prime}\right)=u^{\mu v_{j}} F\left(u^{\mu}\right)=b\left(t_{j}^{\prime}\right)\left(u^{\mu}\right)$

Autrement dit, si $\tilde{v}$ est la valuation de $\mathbb{C}\left\{\tau, \tau^{\prime}\right\}$ associée à $\tilde{b}$ :

$$
\tilde{v} \circ \psi=\mu v \text {. }
$$

La condition sur $v$ devient, pour $\tilde{v}$ :

$$
\tilde{v}\left(\tau^{\mu}-\tau^{\prime \mu}\right)>\mu \tilde{v}(\tau)=\mu \tilde{v}\left(\tau^{\prime}\right)=\mu v_{0} \text {, et } i l \text { existe une unique racine }
$$

$\mu$-ième de l'unité, $\varepsilon$, telle que $v^{\prime}\left(\tau^{\prime}-\varepsilon \tau\right)=v_{1}>v_{0}$ (lemme VI.1.1). 


\section{DÉFORMATIONS ÉQUISINGULIÈRES}

Notons encore $\tilde{y}_{\varepsilon}$ la valuation de $C_{\left\{\tau, \tau^{\prime}\right\}}: \tilde{v}_{\varepsilon}(h)=v_{\tau}(h(\tau, \varepsilon \tau))$ :

on a alors $\tilde{v}_{\varepsilon} \circ \psi\left(f_{i}\left(t_{i}\right)-f_{j}\left(t_{j}^{\prime}\right)\right)=m_{i, j, \varepsilon}(f)$.

Reprenons maintenant la démonstration du théorème VI.2.2 : la condition (b) est également nécessaire puisqu'elle exprime la condition $\left(P_{i, j}\right)$ pour les valuations $v_{\varepsilon}=\tilde{v}_{\varepsilon}$ o $\psi$ de $\mathbb{C}\left\{t_{i}, t_{j}^{\prime}\right\}$.

Pour la réciproque nous avons besoin du lemme :

\section{LEMME VI.2.3 :}

Soit $v$ une valuation de $C\left\{t_{i}, t_{j}^{\prime}\right\}$ satisfaisant à la condition du zème cas, $\tilde{v}$ la valuation de $\left\{\left\{\tau, \tau^{\prime}\right\}\right.$ associée, et $\varepsilon$ l'unique racine $\mu$-ième de l'unité pour laquelle $\tilde{v}\left(\tau^{\prime}-\varepsilon \tau\right)>\tilde{v}\left(\tau^{\prime}\right)=\tilde{v}(\tau)=v_{0}$.

On a alors $\mu v\left(I_{A}^{(i, j)}\right)=\inf \left(m_{i, j, \varepsilon} v_{0}, \tilde{v}\left(\tau^{\mu}-\tau^{\prime \mu}\right)\right)$.

\section{PREUVE DU LEMME :}

Pour tout $f \in \bar{A}, i l$ existe des germes $U$ et $g$ de $\left\{\left\{\tau, \tau^{1}\right\}, U\right.$ inversible, tels que :

(1) $\psi\left(f_{i}\left(t_{j}\right)-f_{j}\left(t_{j}^{\prime}\right)\right)=\tau^{m_{i, j, \varepsilon}(f)} U+\left(\tau^{\prime}-\varepsilon \tau\right) g\left(\tau, \tau^{\prime}\right)$

Lorsque $f \in \tilde{A}_{1} \oplus \ldots \oplus \tilde{A}_{r}, g\left(\tau, \tau^{\prime}\right) \in\left(\tau, \tau^{\prime}\right)^{\mu-1}$ et par conséquent :

$(2)_{\mu} v\left(f_{i}\left(t_{i}\right)-f_{j}\left(t_{j}^{\prime}\right)\right)=\tilde{v}\left(\psi\left(f_{j}\left(t_{j}\right)-f_{j}\left(t_{j}^{\prime}\right)\right) \geq \inf \left(m_{i, j, \varepsilon}(f) v_{0}, \tilde{v}_{i}^{\prime} \tau^{\mu}-\tau^{\prime \mu}\right)\right)$

Donc en particulier :

$\mu v\left(I_{A}^{(i, j)}\right)=\mu \inf \left\{v\left(f_{j}\left(t_{j}\right)-f_{j}\left(t_{j}^{\prime}\right)\right) ; f \in A\right\} \geq \inf \left(m_{i, j, \varepsilon}(f) v_{0}, \tilde{v}\left(\tau^{\mu}-\tau^{\prime \mu}\right)\right)$

On en déduit l'égalité du lemme car il est clair que

$\tilde{v}\left(\tau^{\mu}-\tau^{\prime \mu}\right)=\mu v\left(t_{i}^{\nu}-t_{j}^{\nu^{\nu} j}\right) \geq \mu v\left(I_{A}^{(i, j)}\right)$ et $i 1$ existe $f \in A$ tel que $m_{i, j, \varepsilon}(f)$

$=m_{i, j, \varepsilon} ;$ donc, si $m_{i, j, \varepsilon} v_{0}<\tilde{v}\left(\tau^{\mu}-\tau^{\prime \mu}\right)$,

$\mu v\left(I_{A}^{(i, j)}\right) \leq \mu v\left(f_{i}\left(t_{i}\right)-f_{j}\left(t_{j}^{\prime}\right)\right)=m_{i, j, \varepsilon} v_{0}$ d'après (1). 
PREUVE DE (a) et $(b) \Longrightarrow f \in \AA$ :

D'après $(a), f \in \AA_{1} \oplus \ldots \otimes \AA_{r}$.

L'inégalité $m_{i, j, \varepsilon}(f) \geq m_{i, j, \varepsilon}>0$ entraine $f_{i}(0)=f_{j}(0)$ et la condition $\left(P_{i, j}\right)$ est satisfaite pour les valuations du ler cas.

Dans le 2ème cas on a, d'après le lemme VI.2.3, (2) et (b) :

$\mu v\left(f_{j}\left(t_{j}\right)-f_{j}\left(t_{j}^{\prime}\right)\right) \geq \inf \left(m_{i, j, \varepsilon}(f) v_{0}, v\left(\tau^{\mu}-\tau^{\prime \mu}\right)\right)$

$$
\begin{aligned}
\prime \prime & \geq \inf \left(m_{i, j, \varepsilon} v_{0}, v\left(\tau^{\mu}-\tau^{\prime \mu}\right)\right) \\
\prime \quad & \geq \mu v\left(I_{A}^{(i, j)}\right) .
\end{aligned}
$$

\section{§ 3. SATURATION DES COURBES PLANES ET PROJECTIONS PLANES GÉNEERIQUES.}

Nous reprenons les notations du chapitre IV.

PROPOSITION VI.3.1.:

Il existe un ouvert de Zariski $V$ de $\mathscr{G}$ tel que, si $H \in V, A^{\prime}=\Pi_{H^{*}}^{*}(A)$ a le

même saturé que $A$. ( $A^{\prime}$ désigne l'algèbre de la projection $\Pi_{H}(C)$ ).

PREUVE :

Il existe un ouvert de Zariski $V^{(i, j)}$ de $\mathscr{G}$ tel que, si $H \in V^{(i, j)}, l$ idéal de définition $I_{A}^{(i, j)}$ de $\mathbb{C}\left\{t_{j}, t_{j}^{\prime}\right\}$ qui est engendré par

$\left\{\omega_{k}=z_{k, j}\left(t_{j}\right)-z_{k, j}\left(t_{j}^{\prime}\right) ; k=1, \ldots, N\right\}$ ait même multiplicité que l'idéal engendré par $\left\{a_{1} \omega_{1}+\ldots a_{i v} \omega_{N}, b_{1} \omega_{1}+\ldots+b_{N} \omega_{N}\right\} \quad$ (H étant défini par les équations $\left.a_{1} z_{1}+\ldots a_{N} z_{N}=b_{1} z_{1}+\ldots+b_{N} z_{N}=0\right)$ c'est à dire $I_{A^{\prime}}^{(j, j)}$. Il en résulte, d'après $[R]$, que $I_{A}^{(j, j)}$ et $I_{A^{\prime}}^{(j, j)}$ ont même cloture intégrale et donc, si $H \in V=\bigcap_{i, j} V^{(i, j)}, A$ et $A^{\prime}$ ont même saturé.

On se propose maintenant de démontrer un résultat pour un germe de courbe plane $C$; de ce résultat et de la proposition précédente on déduit aussitôt le théorème VI.0.2. Dorénavant la courbe $C$ est donc supposée plane. 


\section{DÉFORMATIONS ÉQUISINGULIÈRES}

\section{-PROPOSITION VI.3.2.}

Soit A l'anneau local d'un germe de courbe plane réduite $C$. Son saturé Å est déterminé (à isomorphisme analytique près) par les exposants caractéristiques de ses branches $\left(\left[z_{1}\right],\left[z_{8}\right]\right)$ et par les multiplicités d'intersection $m_{i, j}=\left(C_{i} ; C_{j}\right)$ des branches deux à deux.

Inversement la donnée de $\AA$ détermine ces invariants numériques.

Remarquons que ce résultat implique que 1 'ouvert $V$ de la proposition VI.3.1 n'est autre que l'ouvert $\Omega$ des directions de projections génériques de $C$.

\section{LEMME VI.3.3 :}

La proposition VI.3.2 est vraie lorsque $C$ est irréductible.

PREUVE :

Soit $\left(x=t^{\beta_{0}}, y=\sum_{p \geq \beta_{0}}^{\sum} a_{p} t^{p}\right)$ la normalisation de $C$ et $\left\{\beta_{0}, \beta_{1}, \ldots, \beta_{g}\right\}$ les exposants caractéristiques de $C$ définis par les formules de récurrence :

$\beta_{\mathbf{j}}=\inf \left\{k \in \mathbb{N} ; a_{k} \neq 0\right.$ et $e_{i-1}=\operatorname{pgcd}\left(\beta_{0}, \ldots, \beta_{j-1}\right)$ ne divise pas $\left.k\right\}$

On a $: \widetilde{E(A)}=\left\{\left\{\beta_{0}\right\} \cup E_{\boldsymbol{X}}(y)\right\}^{2}=\left\{\beta_{0}, \ldots, \beta_{g}\right\}^{2}$ et $\left\{\beta_{0}, \ldots, \beta_{g}\right\}$

coincide avec la suite caractêristique de $A$ qui détermine $\tilde{A}$ à isomorphisme près (théorème VI.1.6).

Inversement $\widetilde{E}(A)$ est le semi-groupe de $\tilde{A} \subset \mathbb{C}\{t\}$, donc est un invariant analytique de $\mathcal{A}^{\prime}\left(\left[T_{3}\right]\right) ; i 1$ en est donc de même de $\left\{\beta_{0}, \ldots, \beta_{g}\right\}$.

Dans le cas réductible, soit $\left(x_{i}=t_{i}{ }^{\nu_{i}}, y_{i}=\sum_{p \geq v_{i}} \alpha_{p}^{i} t_{i}^{p}\right)$ la normalisation de la $i$-ème branche de $C(i=1, \ldots, r)$ et $\varphi_{j}\left(x_{i}\right)=\tau^{\mu} ; \varphi_{j}\left(y_{j}\right)=\sum_{k \geq \mu} a_{k}^{i} \tau^{k}$.

Remarquons que la suite caractéristique de $\left\{\varphi_{j}\left(x_{j}\right), \varphi_{j}\left(y_{j}\right)\right\}$ est exactement le produit par $\mu_{i}$ de la suite caractéristique de $A_{j}$. D'autre part, d'après la définition VI.2.1, pour deux branches distinctes $C_{i}$ et $C_{j}$, $m_{i, j, \varepsilon}=v_{\tau}\left(\varphi_{j}\left(y_{j}\right)(\tau)-\varphi_{j}\left(y_{j}\right)(\varepsilon \tau)\right)=\inf \left\{k ; a_{k}^{i} \neq \varepsilon^{k} a_{k}^{j}\right\}$. 
Les nombres $m_{i, j, \varepsilon}$ mesurent la distance entre deux feuilles des revêtements ramifiés $C_{i}$ et $C_{j}$ au-dessus de l'axe des $x$ :

LEMME VI.3.4. :

La multiplicité d'intersection $m_{i, j}$ de deux branches $C_{i}$ et $C_{j}$ est donnée, en fonction des $\varepsilon$-valuations de $A$, par :

$$
m_{i, j}=\frac{1}{\mu_{i} \mu_{j}} \sum_{\varepsilon^{\mu=1}} m_{i, j, \varepsilon}
$$

PREUVE :

Soit $v_{x}$ la valuation naturelle de $C\{x\} \subset C\{\tau\}$.

On a :

$$
\begin{aligned}
& m_{i, j}=v_{x}\left(\sum_{\varepsilon}^{\nu_{i}=\nu_{j}^{\nu}=1}{ }^{\nu_{j}}\left(\varepsilon^{\mu_{i}}\right)-y_{j}\left(n \tau{ }^{\mu_{j}}\right)\right) \\
& m_{i, j}=\frac{1}{\mu \mu_{i}{ }_{j}} \sum_{\varepsilon^{\mu}=\eta^{\mu}}=1 v_{\tau}\left[y_{i}\left((\varepsilon \tau)^{\mu}\right)-y_{j}\left((n \tau)^{\mu} j\right)\right] \\
& m_{i, j}=\frac{1}{\mu \mu_{i} \mu_{j}} \sum_{\varepsilon^{\mu}} \sum_{=\eta^{\mu}=1} m_{i, j, \eta \varepsilon}=\frac{1}{\mu_{i} \mu_{j}} \sum_{\varepsilon^{\mu}=1} m_{i, j, \varepsilon}
\end{aligned}
$$

Nous allons préciser la valeur des nombres $m_{i, j, \varepsilon}$ lorsque $\varepsilon$ décrit les racines $\mu$-ème de l'unité, pour deux branches distinctes $c_{j}$ et $C_{j}$. Notons $\left\{\beta_{0}^{i}=\mu, \beta_{1}^{\mathfrak{i}}, \ldots, \beta_{g_{j}}^{i}\right\}$ la suite caractéristique de $\varphi_{j}\left(A_{j}\right)$ : c'est le produit par $\mu_{i}$ de la suite des exposants caractéristiques de $C_{j}$. Nous supposons alors que les suites caractéristiques de $\varphi_{j}\left(A_{i}\right)$ et $\varphi_{j}\left(A_{j}\right)$ coîncident jusqu'à l'ordre w inclu et nous noterons dorénavant :

$$
\left\{\beta_{0}=\mu, \beta_{1}=\beta_{1}^{i}=\beta_{1}^{j}, \ldots, \beta_{W}=\beta_{W}^{i}=\beta_{W}^{j}\right\}
$$

et $e_{s}=\operatorname{pgcd}\left(\beta_{0}, \ldots, \beta_{s}\right)$, pour $0 \leq s \leq w$.

Enfin soit $\gamma_{i, j}=\sup \left\{m_{i, j, \varepsilon} ; \varepsilon^{\mu}=1\right\}$. 


\section{DÉFORMATIONS ÉQUISINGULIÈRES}

\section{LEMME VI.3.5. :}

Il existe un entier $q \leq w$ tel que $l$ 'ensemble des entiers $\left\{m_{i, j, \varepsilon} ; \varepsilon^{\mu}=1\right\}$ soit égal à $\left\{\beta_{1}, \beta_{2}, \ldots, \beta_{q}, \gamma_{i j}\right\}$ pour $q \geqslant 1$ et à $\left\{\gamma_{i, j}\right\}$ pour $q=0$.

De plus, le nombre de racines $\mu$-ème de l'unité pour lesquelles $m_{i, j, \varepsilon}=\beta_{S}$ (resp. $m_{i, j, \varepsilon}=\gamma_{i, j}$ ) est égal à $e_{s-1}-e_{s}$ pour $s=1, \ldots, q\left(\right.$ resp. $\left.e_{q}\right)$.

\section{PREUVE :}

Soit $\underline{\varepsilon}$ satisfaisant à $m_{i, j, \underline{\varepsilon}}=\gamma_{i, j}$; pour tout $k<\gamma_{i, j}, a_{k}^{i}=(\underline{\varepsilon})^{k} a_{k}^{j}$.

On définit l'entier q par les conditions suivantes :

$$
\begin{aligned}
& q=0 \text { si } \gamma_{i, j} \leq \beta_{1} \\
& \operatorname{sinon} \beta_{q}<\gamma_{i, j} \leq \beta_{q+1}\left(\text { en posant } \beta_{w+1}=\infty\right) .
\end{aligned}
$$

Prenons alors $\varepsilon$ tel que $m_{i, j, \varepsilon}<\gamma_{i, j}: m_{i, j, \varepsilon}=\inf \left\{k ; a_{k}^{j} \neq \varepsilon^{k} a_{k}^{j}\right\}$.

Or $m_{i, j, \varepsilon}<\beta_{q+1}$ implique que $e_{q}$ divise $m_{i, j, \varepsilon}$ et donc $\varepsilon^{e^{q}} \neq(\underline{\varepsilon})^{e} q$. Comme pour tout entier $s \geq 1$, $e_{s}$ divise $e_{s-1}$ et que $\varepsilon^{e_{0}}=(\underline{\varepsilon})^{e_{0}}=1$, il existe un entier $t$ pour lequel $\varepsilon^{e_{t}} \neq(\underline{\varepsilon})^{e_{t}}$ et ${ }_{\varepsilon}{ }^{e_{t-1}}=(\underline{\varepsilon})^{e_{t-1}}$. On en déduit que $\mathrm{m}_{\mathbf{i}, \mathbf{j}, \varepsilon}=\beta_{\mathrm{t}}$ puisque $a_{\beta_{t}}^{\mathbf{j}} \neq 0, \varepsilon^{\beta_{t}} \neq(\underline{\varepsilon})^{\beta}$, et pour $k<\beta_{t}$ et $a_{k}^{i} \neq 0$ $e_{t-1}$ divise $k$ et par conséquent $\varepsilon^{k}=(\varepsilon)^{k}$.

La condition $\varepsilon^{e} e_{t-1}=(\underline{\varepsilon})^{e} e_{t-1}$ et ${ }_{\varepsilon}{ }^{e} \neq(\underline{\varepsilon})^{e}$ t , est nécessaire et suffisante pour que $m_{i, j, \varepsilon}=\beta_{t}$, ce qui prouve la seconde partie du lemme.

\section{PREUVE DE LA PREMIËRE PARTIE DE LA PROPOSITION VI.3.2. :}

D'après les deux lemmes précèdents :

(*) $\mu_{i} \mu_{j} m_{i, j}=\left(e_{0}-e_{1}\right) \beta_{1}+\ldots+\left(e_{q-1}-e_{q}\right) \beta_{q}+e_{q} \gamma_{i, j}$

Introduisons encore $e_{i-1}=n_{j} e_{j}$ et

$\bar{\beta}_{s}=\left(n_{1}-1\right) n_{2} \ldots n_{s-1} \beta_{1}+\ldots+\left(n_{s-1}-1\right) \beta_{s-1}+\beta_{s}$. 
Les nombres $\left\{\bar{\beta}_{S} ; 0 \leq s \leq q\right\}$ sont les premiers générateurs minimaux des semi-groupes de $\varphi_{i}\left(A_{j}\right)$ ou $\varphi_{j}\left(A_{j}\right)$ et on a :

$\bar{\beta}_{S+1}=n_{S} \bar{\beta}_{S}-\beta_{S}+\beta_{S+1} \cdot\left(\left[Z_{8}\right]\right.$ théorème 3.9).

On peut alors écrire:

$\mu_{i} \mu_{j} m_{i, j}=e_{q-1} \bar{\beta}_{q}-e_{q} \beta_{q}+e_{q} \gamma_{i, j}$

D' où

$e_{q-1} \bar{\beta}_{q}<\mu_{j} \mu_{j} m_{i j} \leq e_{q}\left(n_{q} \bar{\beta}_{q}-\beta_{q}+\beta_{q+1}\right)=e_{q} \bar{\beta}_{q+1}$

Ainsi l'entier q est déterminé par la multiplicité d'intersection $m_{i, j}$ et les exposants caractéristiques des branches $c_{i}$ et $c_{j}$, ce qui, d'après le lemme VI.3.5 et le théorème VI.2.2 démontre la première partie de la proposition VI.3.2.

\section{LEMME VI.3.6. :}

Soit $f \epsilon_{i=1}^{r} \tilde{A}_{i} \subset \bar{A}$ et $\underline{\varepsilon}$ tel que $m_{i, j, \varepsilon}=\gamma_{i, j}$. Si $m_{i, j, \underline{\varepsilon}}(f)>m_{i, j, \underline{\varepsilon}}$, pour toute racine $\mu$-ème de l'unité on $a: m_{i, j, \varepsilon}(f) \geq m_{i, j, \varepsilon}$.

\section{PREUVE :}

Notons $\varphi_{j}\left(f_{i}\right)=\Sigma b_{k}^{i} \tau^{k} ;$ la condition $m_{i, j, \underline{\varepsilon}}(f) \geq m_{i, j, \underline{\varepsilon}}$ équivaut à

$b_{k}^{i}=(\varepsilon)^{k} b_{k}^{j}$ pour tout entier $k<\gamma_{i, j}$.

Distinguons deux cas :

1) Si $m_{i, j, \varepsilon}=\beta_{t}<\gamma_{i, j}$ on a $\varepsilon^{e_{t-1}}=(\underline{\varepsilon})^{e_{t-1}}$ et

$m_{i, j, \varepsilon}(f) \geq \inf \left\{k \in\left\{\beta_{0}, \beta_{1}, \ldots, \beta_{q}\right\}^{2} ; e_{t-1}\right.$ ne divise pas $\left.k\right\}=\beta_{t}$ car la condition $f_{i} \in \tilde{A}_{i}$ entraine, pour $b_{k}^{i} \neq 0, k \in\left\{\beta_{0}^{i}, \ldots, \beta_{g_{i}}^{i}\right\}^{2}$, et donc $k \in\left\{\beta_{0}, \beta_{1} \ldots, \beta_{q}\right\}^{2}$ pour $k<\gamma_{i, j}$. 
2) Si $m_{i, j, \varepsilon}=\gamma_{i, j}, \quad \varepsilon^{e_{q}}=(\underline{\varepsilon})^{e_{q}}$ et on déduit de même $m_{i, j, \varepsilon}(f) \geq \gamma_{i, j}=m_{i, j, \varepsilon}$ ce qui termine la démonstration du lemme.

\section{LEMME VI.3.7. :}

Soit $\Pi_{j}: \bar{A}=\bar{A}_{1} \oplus \ldots \oplus \bar{A}_{r} \longrightarrow \bar{A}_{j}$ la projection canonique sur le $i$-ème facteur. On a $\AA_{i}=\pi_{j}(\tilde{A})$.

\section{PREUVE :}

Commençons par démontrer, pour trois indices $i, j, k$ l'inégalité triangulaire : $\gamma_{j, k} \geq \inf \left(\gamma_{i, j}, \gamma_{i, k}\right)$ et $\gamma_{j, k}=\gamma_{i, j}$ si $\gamma_{i, j}<\gamma_{i, k}$.

a) Il existe des racines $\mu$-ème de l'unité, $\varepsilon$ et $\varepsilon^{\prime}$ telles que pour tout entier $p<\inf \left(\gamma_{i, j}, \gamma_{i, k}\right)$ on ait :

$a_{p}^{i}=\varepsilon^{p} a_{p}^{j}$ et $a_{p}^{i}=\varepsilon^{\prime p} a_{p}^{k}$. Et donc $a_{p}^{j}=\left(\frac{\varepsilon^{\prime}}{\varepsilon}\right)^{p} a_{p}^{k}$.

On en déduit $\gamma_{j, k} \geq \inf \left(\gamma_{i, j}, \gamma_{i, k}\right)$

b) Si $\gamma_{i, j}<\gamma_{i, k}$, pour toute racine $\mu$-ème de l'unité $\varepsilon$, il existe un entier $p \leq \gamma_{i, j}$ tel que $a_{p}^{i} \neq \varepsilon^{p} a_{p}^{j}$ et $i l$ existe une racine $\mu$-ème $\varepsilon^{\prime}$ telle que $a_{p}^{i}=\varepsilon^{\prime p} a_{p}^{k}$. Donc $a_{p}^{j} \neq\left(\frac{\varepsilon^{\prime}}{\varepsilon}\right)^{p} a_{p}^{k}$ : pour toute racine $\varepsilon^{\prime \prime}=\frac{\varepsilon^{\prime}}{\varepsilon}$ de l'unité, $i 1$ existe donc $p \leq \gamma_{i, j}$ vérifiant $a_{p}^{j} \neq \varepsilon^{\prime \prime} p a_{p}^{k} c^{\prime}$ est à dire $\gamma_{j, k} \leq \gamma_{i, j}$. Démontrons maintenant le lemme : soit $h_{i} \in \tilde{A}_{i}$ et $\varphi_{i}\left(h_{i}\right) \underset{p \geq 0}{=\Sigma} c_{p}^{i}{ }^{p}$. Choisissons, pour tout indice $j$ distinct de $i$, une racine $\mu$-ème de l'unité $\varepsilon_{i, j}$, telle que $m_{i, j, \varepsilon_{i, j}}=\gamma_{i, j}$ et définissons :

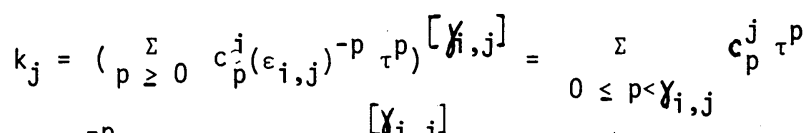

avec $c_{p}^{j}=c_{p}^{i}\left(\varepsilon_{i, j}\right)^{-p}$, le symbole $\left(j^{\left[\gamma_{j, j}\right]}\right.$ signifiant que l'on a tronqué la série à l'ordre $\gamma_{i, j}$. 
Pour $p<\gamma_{i, j}$ et $c_{p}^{i} \neq 0$, soit $s$ défini $\operatorname{par} \beta_{s} \leq p<\beta_{s+1} ; p$ est multiple de $e_{s}=\operatorname{pgcd}\left(\beta_{0}, \beta_{1}, \ldots, \beta_{s}\right)$, donc multiple de $\mu_{i}$ et $\mu_{j}$; on peut donc trouver $h_{j} \in \AA_{j}$ tel que $k_{j}=\varphi_{j}\left(h_{j}\right): h_{j}=\sum_{0 \leq p<\gamma_{i, j}} c_{p}^{j} t_{j}^{p / \mu_{j}}$.

Il nous reste à vérifier que $h=h_{1} \oplus \ldots \oplus h_{r}$ vérifie les conditions $P_{j, k}$ pour avoir $h \in \hat{A}$ et $\pi_{i}(h)=h_{i}$. Pour tout $\varepsilon$ et tout entier $p$ :

$$
c_{p}^{k} \varepsilon^{p}-c_{p}^{j}=c_{p}^{i} \varepsilon_{i, j}^{-p} \quad\left[\left(\varepsilon_{i j} \varepsilon_{i k}^{-1} \varepsilon\right)^{p}-1\right] .
$$

On suppose $\gamma_{i, j} \leq \gamma_{i, k}$.

Soit $p<\gamma_{i, j}$ tel que $c_{p}^{i} \neq 0$; les trois suites caractéristiques cọincident jusqu'à l'ordre $q,\left\{\beta_{0}, \ldots, \beta_{q}\right\}$ et $p$ est multiple de $e_{s}$ si $\beta_{s} \leq p<\beta_{s+1}$.

Choisissons $\varepsilon=\varepsilon_{j, k}$; on a $\left(\varepsilon_{i j}\right)^{e_{s}}=\left(\varepsilon_{i k}\right)^{e_{s}}=\left(\varepsilon_{j, k}\right)^{e_{s}^{s}}=1$

et donc $m_{j, k, \varepsilon_{j, k}}(h) \geq \gamma_{i, j}$.

Lorsque $\gamma_{i, j}<\gamma_{i k}, \gamma_{i, j}=\gamma_{j, k}$ et d'après le lemme VI.3.6 la condition $P_{j, k}$ est vérifiée pour $h$. Lorsque $\gamma_{i, j}=\gamma_{i, k}$, puisque les séries $\varphi_{j}\left(h_{j}\right)$ et $\varphi_{k}\left(h_{k}\right)$ sont tronquées à partir de cet ordre, la condition est automatiquement satisfaite $\operatorname{car} m_{j, k, \varepsilon_{j, k}}(h)=+\infty$.

REMARQUE :

On déduit facilement du lemme VI.3.7. que si le couple $\left(h_{i}, h_{j}\right) \in \tilde{A}_{i} \oplus \tilde{A_{j}}$ vérifie les conditions $P_{i, j}$, il existe $h \in \tilde{A}$ tel que $\pi_{j}(h)=h_{i}$ et $\pi_{j}(h)=h_{j}$. En effet, soit $H \in \hat{A}$ construit à partir de $h_{j}$ comme dans le lemme précédent : $\Pi_{j}(H)=h_{i}$ et $\Pi_{j}(H)$ coïncide avec $h_{j}$, jusqu'à l'ordre $\gamma_{i, j}$.

De même en appliquant la même construction à partir de $h_{j}-\Pi_{j}(H)$ on obtient $K \in \tilde{A}$ tel que $\Pi_{j}(K)=h_{j}-\Pi_{j}(H)$ et $\Pi_{j}(K)=0$.

Donc $\pi_{j}(H+K)=h_{i}$ et $\pi_{j}(H+K)=h_{j}$. 
FIN DE LA DÉMONSTRATION DE LA PROPOSITION VI.3.2. :

D'après le lemme VI.3.7 $\tilde{A}_{j}=\pi_{j}\left(\AA^{\prime}\right)$ et les exposants caractéristiques

$\left\{\beta_{0}^{i}, \beta_{1}^{i}, \ldots, \beta_{g_{j}}^{i}\right\}$ sont des invariants analytiques de $\AA_{i}$ (lemme VI.3.3) donc de $\AA$.

D'autre part, d'après la dernière remarque ci-dessus on a :

$\operatorname{dim}_{\mathbf{C}}\left[\left(\AA_{i} \odot \AA_{j}\right) /\left[\pi_{i} \oplus \pi_{j}\right](\tilde{A})\right]=\#\left\{p \in\left\{\beta_{0}, \ldots, \beta_{q}\right\}^{2} ; p<\gamma_{i, j}\right\}$

ce qui montre que $\gamma_{i, j}$ est un invariant analytique de $\tilde{A}$, donc auss $i m_{i, j}$ d'après la formule (*). 
[Bo]

J. BOARDMAN. "Singularities of differential mappings" Publ. I.H.E.S.

$n^{\circ} 33$ (1967).

[Br] K. BRAUNER. Zur Geometrie der Funktionen Zweir komplexen Veränderlichen. Abh. Math. Sem. Hamburg 6 (1928) 1.54.

$[B-S]$

J. BRIANÇON et J.P. SPEDER. "Les conditions de Whitney impliquent $\mu^{*}$ constant" Annales de l'Inst. Fourier (26. fasc. 2) (1976).

[ Bu]

R.0. BUCHIJEISS "On deformations of monomial curves" in Séminaire sur les singularités de surfaces. Publ. centre de Math. de l'Ecole Polytechnique (1976-77).

$[B-G]$

R.0. BUCHWEISS et G.M. GREUEL. "Le nombre de Milnor, équisingularité et déformations des singularités de courbes réduites" Preprint (Juillet 78).

[D]

C. DELORME. "Sous monoides d'intersection complète de $\mathbb{N}$ "

An. Scient. Ec. Norm. Sup. 4ème série tome 9 (1976).

$\left[\mathrm{Gi}_{1}\right]$

M. GIUSTI. "Sur les singularités d'intersections complètes quasi homogènes" Ann. inst. Fourier Tome 27 fasc. 3 (1977).

$\left[\mathrm{Gi}_{2}\right]$

M. GIUSTI. Classification des singularités isolées

d'intersections complètes Publ. Centre de Math de

l'Ecole Polytechnique. Palaiseau (1977).

[G - F] H. GRAUERT et K. FRITZCHE "Several Complex variables"

Springer Verlag éditeur (1976).

[G - H] G.M. GREUEL et H. HAMM "Invarianten quasihomogener vollständiger Durchschnitte" Preprint.

[ $\left.\mathrm{H}_{1}\right]$ H. HIRONAKA. "Stratification and flatness" Lectures at the Nordic Summer School 0slo (1976) Noordhoff. Per Holm éditeur.

[ $\left.\mathrm{H}_{2}\right]$ H. HIRONAKA "Normal cones in analytic Whitney stratifications"

Publ. Math. I.H.E.S. (36) (1969). 
F. KUNZ. "The value semigroup of a one-dimensional Gorenstein ring". Proc. A.M.S. vol.25 (1970).

[Lế

Lê D.T. "Calcul du nombre de Milnor d'une singularité isolée d'intersection complète" Publ. du Centre de Math. de l'Ecole Polytechnique (1973) ou Funct. Anal. i ego Prilo (8) (1974) p.45-49.

[Lê. ${ }_{2}$ Lê D.T. "Sur un critère d'équisingularité". Sëminaire Norguet 1970-71. Springer Lectures Notes $n^{\circ}$ 409. Cf. aussi : même titre, note aux C.R.A.S. Paris t. 272 (1971).

[L - R ] Lê. D.T. et C.P.RAMANUJAM. "The invariance of the Milnor number implies the invariance of the topological type". Amer. J. of Math. 98, 1 (1976). systems conference, Academic Press (1973).

[Mi] J. MILNOR "Singular points of complex hypersurfaces". Ann. Math. Studies $n^{\circ} 61$ Princeton U.P. (1968).

[N] R. NARASIMHAN "Introduction to the theory of analytic spaces" Lecture Notes $n^{\circ} 25$ Springer editeur (1966).

[P - T] F. PHAM et $B$. TEISSIER "Fractions Lipschitziennes d'une algèbre analytique complexe et saturation de Zariski" Publ. Centre de Math. de l'école Polytechnique (1969) ou Actes du Congrès de Math. Nice (1970).

[Pi] H. PINKHAM "Déformation of Algebraic Varieties with $G_{m}$ action" Astérisque vol. 20 (1974). Proc. Camb. Phil. Soc. 57 (1961).

[Sc] M. SHAPS "Déformations de courbes gauches" in Astérisque $n^{\circ} 7$ et 8 (1973). 

$(1960 / 61)$.

[St ] J. STUTZ. "Equisingularity and equisaturation" Amer. Journ. of Math. 94 (1972).

$\left[T_{1}\right] \quad$ B. TEISSIER. "The Hunting of invariants" Lecture at the Nordic Summer School 0slo (1976) Noordhoff. Per Holm éditeur.

$\left[\mathrm{T}_{2}\right]$ B. TEISSIER. "Résolution simultanée I et II "in Séminaire sur les singularités dẹs surfaces, Ecole Polytechnique Palaiseau (1976-77).

$\left[T_{3}\right] \quad$ B. TEISSIER. Appendice à $\left[Z_{8}\right]$.

$\left[\mathrm{T}_{4}\right] \quad$ B. TEISSIER. "Cycles évanescents sections planes et conditions de. Whitney" in Singularités à Cargèse. Astérisque $n^{\circ} 7$ et 8 (1973).

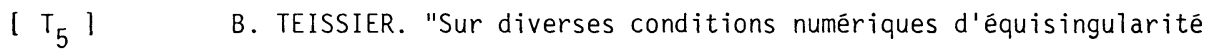
des familles de courbes". Publ. du centre de Math. de l'Ecole Polytechnique (1975).

[ Th ] R. THOM. "Ensembles et morphismes stratifiés" Bull. Amer. Math. Soc. 75 (1969).

[ Wa ] J. WAHL. "Equisingular deformations of plane algébroid curves". Trans. of the A.M.S. (Providence R.I.) vol. 193 (1974).

[ W I H. WHITNEY. "Tangents to analytic variety" Ann. of Math. (81) (1965).

$\left[z_{1}\right] \quad 0$. ZARISKI. "Studies in equisingularity I" Am. Journal of Math. 87 (1965).

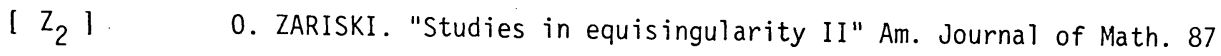
(1965).

$\left[\mathrm{Z}_{3}\right] \quad 0$. ZARISKI. "Studies in equisingularity III" Am. Journal of Math. 90 (1968). 


\section{RÉFÉRENCES}

$\left[\mathrm{Z}_{4}\right]$ 0. ZARISKI. "General theory of saturation and of satured local rings II" American Journal of Math. 93 (1971).

$\left[Z_{5}\right] \quad 0$. ZARISKI. "on the topology of Algebroid Singularities" Am. Journal of Math 54 (1932).

$\left[Z_{6}\right] \quad 0$. ZARISKI. "Contributions to the problem of equisingularity" in : Questions on algebraic varieties. C.I.M.E. (III Ciclo 1969).

$\left[z_{7}\right]$ 0. ZARISKI. "Some open questions onthe theory of singularities" Bull. A.M.S. 77-4 (1971).

$\left[Z_{8}\right] \quad 0$. ZARISKI. "Modules des branches planes". Publ. du centre de Math. de l'Ecole Polytechnique (1973). 